\title{
A Strategy for Conditional Orthogonal Sequential CuAAC Reactions Using a Protected
}

\section{Aromatic Ynamine}

Marine Z. C. Hatit, Ciaran P. Seath, Allan J. B. Watson, ${ }^{*}$ and Glenn A. Burley*

Department of Pure and Applied Chemistry, WestCHEM, University of Strathclyde, Glasgow, G1 1XL, UK. Email: glenn.burley@strath.ac.uk, allan.watson.100@strath.ac.uk.

\begin{abstract}
A method for conditional control of orthogonal sequential $\mathrm{Cu}$-catalyzed azide alkyne cycloaddition (CuAAC) reactions is reported. The inherent reactivity of an aromatic ynamine is controlled by a silyl protecting group that allows the selective $\mathrm{CuAAC}$ reaction of less reactive alkynes. Alternatively, the same protected ynamine undergoes selective CuAAC reaction via silyl deprotection in situ to give the ynamine click products. This allows complete orthogonal control of dialkyne systems and provides a unifying strategy for chemoselective CuAAC ligations in multi-alkyne/azide systems.
\end{abstract}

The Cu-catalyzed azide-alkyne cycloaddition (CuAAC) or 'click' reaction has become a method of choice for a variety of broadly different applications throughout both chemistry and biology. ${ }^{1,2}$ This popularity arises from the reliable synthesis of 1,4-substituted triazoles using readily available terminal alkyne and azides. The predictability of this reaction has led to broad utility within materials chemistry, ${ }^{3}$ chemical biology, ${ }^{4}$ and combinatorial chemistry. ${ }^{5}$ A powerful extension of the CuAAC reaction uses multi-alkyne/azide systems, where several click events are performed sequentially. ${ }^{6}$ This allows the generation of, for example, two unique triazole products using two alkynes and two azides, or multi-functional products from the union of a diyne with two azide partners. The success of this strategy relies upon chemoselective control of the alkyne and azide coupling partners.

Azide chemoselectivity is typically achieved using chelate-direction, where a Cu-chelating site increases the reactivity of an azide in close proximity. ${ }^{7}$ Alkyne chemoselectivity has been the primary 
focus in this area, with two main approaches. (1) Alkyne deactivation: Protected alkynes allow orthogonal control (Scheme 1a). ${ }^{8}$ While this has led to a number of elegant reports of sequential click reactions, the order of reactivity is typically determined at inception with little opportunity for diversification. (2) Alkyne activation: several highly reactive alkynes have been developed to establish a reactivity gradient. ${ }^{9}$ However, truly orthogonal reactivity has only been demonstrated in strainpromoted alkyne-azide cycloaddition (SPAAC)/CuAAC bifunctional systems where the high azidophilicity of cyclic alkynes allows selectivity over terminal alkynes in the absence of a $\mathrm{Cu}$ catalyst (Scheme 1b). ${ }^{10}$ Transient protection of cyclooctynes offers sequential control; ${ }^{11}$ however, the formation of regioisomeric products from SPAAC can pose problems for downstream applications due to potential differences in regioisomer pharmacology. ${ }^{12}$

At present, sequential CuAAC methodologies are uni-directional, i.e., there is a pre-designed deactivation of one alkyne and this reactivity gradient cannot be completely overturned. In addition, few methods are available to perform sequential CuAAC reactions exploiting chemoselective control of both alkyne and azide reagents. ${ }^{13}$ Here we describe a conditional strategy for complete orthogonal control over CuAAC reactions in multi-alkyne/azide systems using a single aromatic ynamine construct (Scheme 1c).

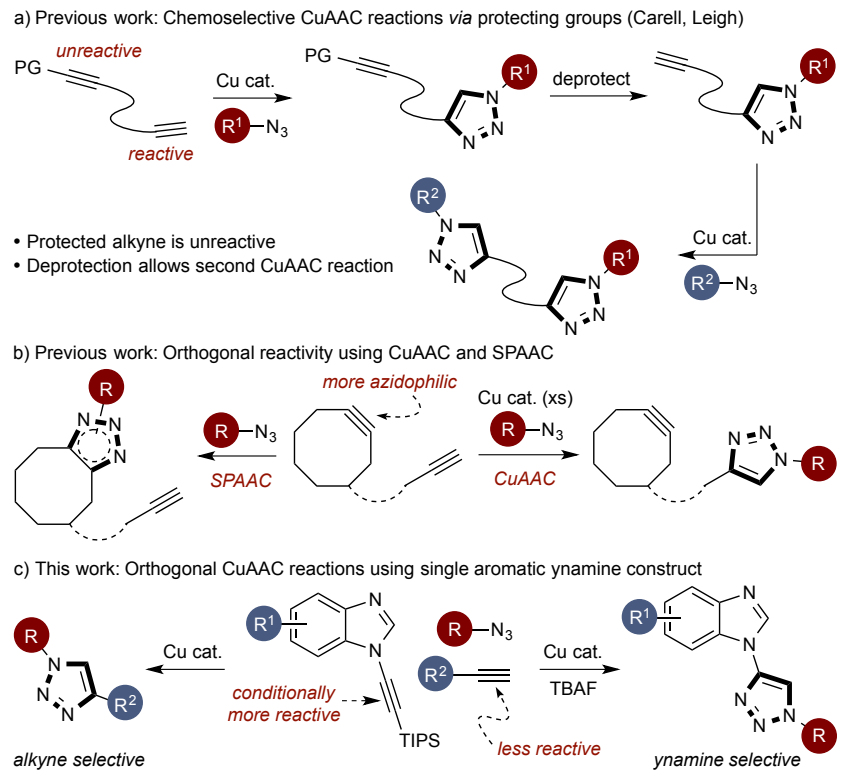


Scheme 1. (a) Alkyne selectivity using protecting groups. (b) Control using CuAAC vs. SPAAC. (c) Orthogonal alkyne/ynamine selectivity using a conditional strategy.

Aromatic ynamines display enhanced reactivity over a series of more conventional alkynes due to a shift in the rate-determining step (RDS) of this class of reagent. ${ }^{14}$ The enhanced reactivity of an ynamine, (e.g., 1) vs. a standard terminal alkyne (e.g., 2a) enables chemoselective triazole formation under CuAAC conditions (Scheme 2). ${ }^{15}$

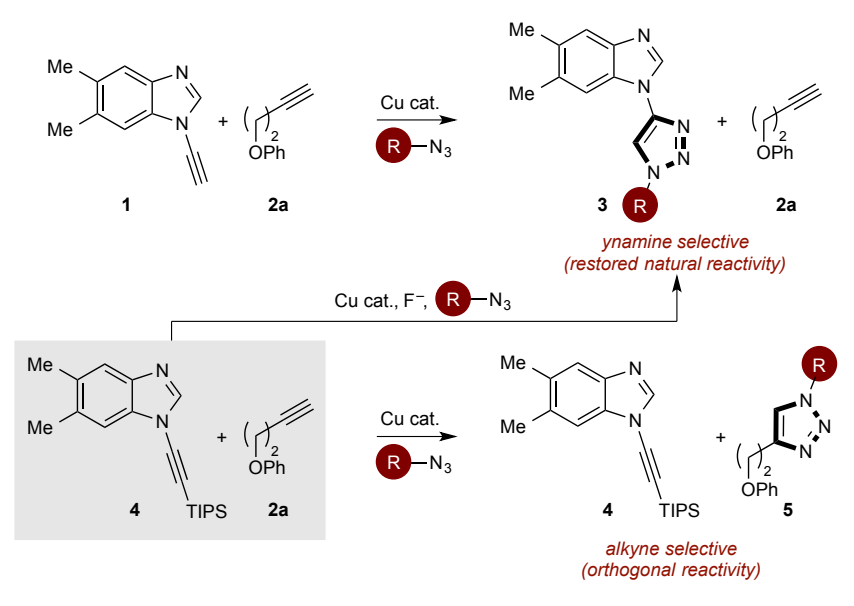

Scheme 2. Design plan.

The reciprocal reaction, i.e., chemoselective reaction at the less reactive alkyne, is rather more difficult based on this kinetic profile. This is another example of uni-directional CuAAC. However, we reasoned that conditional orthogonal control could be delivered using a simple protecting group strategy. Specifically, TIPS protection of $\mathbf{1}$ to give $\mathbf{4}$ would block the natural reactivity of $\mathbf{1}$ and thereby allow the reaction of a less reactive alkyne (e.g., 2a). This would effectively reverse the reactivity trend. To restore the normal reactivity, a rapid in situ silyl deprotection using a fluoride source would deliver 1 that would then react preferentially (Scheme 2). This approach would deliver a conditional bi-directional strategy for sequential control of two CuAAC reactions.

We began our investigation through the reaction of $\mathbf{4}$, benzyl azide $\left(\mathrm{BnN}_{3}, \mathbf{6 a}\right)$, and a representative terminal alkyne (2a) under a range of standard reaction conditions (Table 1). First, chemoselectivity was established for alkyne 2a. Standard CuAAC conditions led to complete selectivity for 2a, 
delivering triazole product 5a in quantitative yield without any degradation of $\mathbf{4}$ (entry 1 ). We then sought to restore ynamine chemoselectivity though addition of TBAF as a source of $\mathrm{F}^{-}$. Initial experiments using standard $\mathrm{Cu}(\mathrm{I})$ ligands (1-(trans-2-hydroxycyclohexyl)-4-(N,Ndimethylaminomethyl)-1,2,3-triazole (AMTC), tris(3-hydroxypropyltriazolylmethyl)amine (THPTA)) and TBAF or polymer-supported fluoride were unsuccessful in any solvent mixture, delivering mixtures of products (entries 2-6). Removal of $\mathrm{Cu}$-ligating additives and variation of the solvent (entries 7-10) led quickly to a system that delivered complete chemoselectivity for 3a (entry 10). Solvent choice was an important factor for chemoselective control. Standard CuAAC solvent mixtures of DMSO $/ \mathrm{H}_{2} \mathrm{O}$ conditions operated well for CuAAC reaction of the alkyl alkyne 2a whereas DMSO and $\mathrm{DMSO} / \mathrm{H}_{2} \mathrm{O}$ mixtures were not effective for selectivity at the ynamine 4. Ynamine selectivity was optimum using $\mathrm{MeCN}$ as the reaction medium. This difference in medium may be attributable to the requirement for sodium ascorbate (NaAsc) as an additive to promote reaction of $\mathbf{2 a}$ and the improved solubility of $\mathrm{NaAsc}$ in $\mathrm{H}_{2} \mathrm{O}$. NaAsc was not necessary for reaction of $\mathbf{4}$. That $\mathbf{4}$ does not need NaAsc as a promoter may be attributable to reduction of $\mathrm{Cu}(\mathrm{II})$ to $\mathrm{Cu}(\mathrm{I})$ via Glaser coupling. ${ }^{2}$ Since complete chemoselectivity for reaction at the ynamine using the in situ deprotection method (entry10), this suggests that the silyl deprotection is sufficiently rapid to have little consequence of the CuAAC process, i.e., the overall rate of silyl deprotection and CuAAC of $\mathbf{4}$ is less than the CuAAC rate of $\mathbf{2 a}$.

Table 1. Reaction development. ${ }^{a}$

\begin{tabular}{|c|c|c|c|}
\hline entry & \multicolumn{2}{|c|}{ reaction conditions } & $\mathbf{3 a}: 5 \mathbf{a}(\%)^{b}$ \\
\hline 1 & \multicolumn{2}{|c|}{$\mathrm{NaAsc}, \mathrm{AMTC}, \mathrm{DMSO} / \mathrm{H}_{2} \mathrm{O}(1: 1)$} & $0: 100$ \\
\hline 2 & \multicolumn{2}{|c|}{ NaAsc, AMTC, TBAF, $\mathrm{MeOH} / \mathrm{H}_{2} \mathrm{O}(1: 1)$} & 47:53 \\
\hline 3 & \multicolumn{2}{|c|}{ NaAsc, AMTC, TBAF, DMSO/ $\mathrm{H}_{2} \mathrm{O}(1: 1)$} & $20: 80$ \\
\hline
\end{tabular}




\begin{tabular}{ccc}
\hline 4 & NaAsc, AMTC, PS Fluoride, DMSO/ $\mathrm{H}_{2} \mathrm{O}$ & $15: 85$ \\
5 & NaAsc, THPTA, TBAF, DMSO/ $\mathrm{H}_{2} \mathrm{O}(1: 1)$ & $43: 57$ \\
6 & NaAsc, THPTA, PS Fluoride, DMSO/ $\mathrm{H}_{2} \mathrm{O}(1: 1)$ & $63: 37$ \\
7 & PS Fluoride, DMSO & $35: 65$ \\
8 & TBAF, DMSO & $47: 53$ \\
9 & TBAF, MeOH & $16: 84$ \\
10 & TBAF, MeCN & $100: 0$ \\
\hline
\end{tabular}

${ }^{a}$ 2a (1 equiv), 4 (1 equiv), 6a (1 equiv), $\mathrm{Cu}(\mathrm{OAc})_{2}(5 \mathrm{~mol} \%), \mathrm{NaAsc} / \mathrm{AMTC} / \mathrm{THPTA}(10 \mathrm{~mol} \%)$, fluoride source (1.1 equiv), solvent $(0.03 \mathrm{M})$, r.t., 16 h. ${ }^{b}$ Determined by HPLC analysis using an internal standard. PS = polymer supported.

With optimized conditions for orthogonal reactivity in this benchmark system established, we progressed to look at the generality of the process. The scope and orthogonality of the developed conditions A (ynamine-selective) and B (alkyne-selective) was first assessed using a series of azide components (Scheme 3). Both sets of conditions proved to be robust, selectively delivering the expected products in high yield regardless of steric or electronic nature of the azide. In addition biorelevant azides were well tolerated, such as the fluorophores $\mathbf{6 g}$-i, and biotinyl azide $\mathbf{6 j}$. 

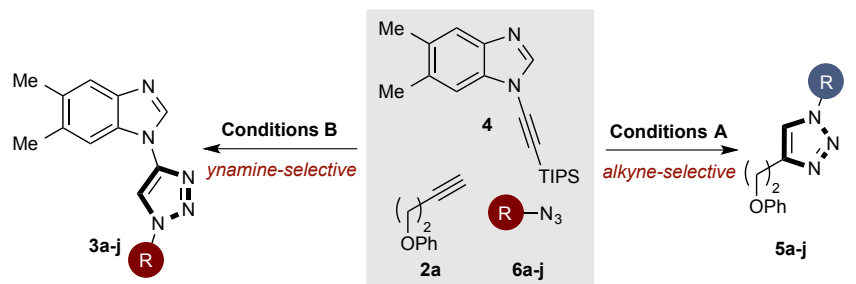

Conditions A: $\mathrm{Cu}(\mathrm{OAc})_{2}(5 \mathrm{~mol} \%)$, NaAsc (10 mol \%), AMTC (10 mol \%), DMSO/ $\mathrm{H}_{2} \mathrm{O}$ (1:1), r.t. Conditions B: $\mathrm{Cu}(\mathrm{OAC})_{2}(5 \mathrm{~mol} \%), \operatorname{TBAF}(1.1$ equiv), $\mathrm{MeCN}$, r.t.

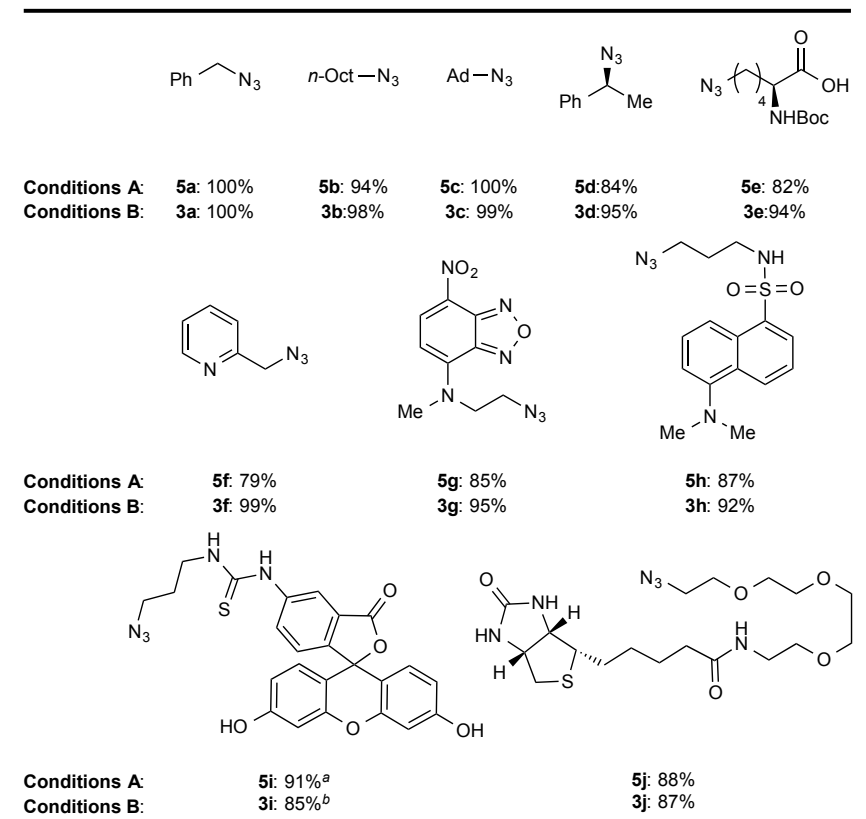

Scheme 3. Selective orthogonal CuAAC reactions of $\mathbf{4}$ and $\mathbf{2 a}$ using a variety of azides using conditions A or B. Isolated yields. ${ }^{a}$ Using $\mathrm{MeOH} / \mathrm{H}_{2} \mathrm{O}(1: 1)$ as solvent. ${ }^{b}$ Using $\mathrm{MeOH}$ as solvent.

The orthogonality of the approach was further probed using a series of different alkyne components vs. 4 (Scheme 4).

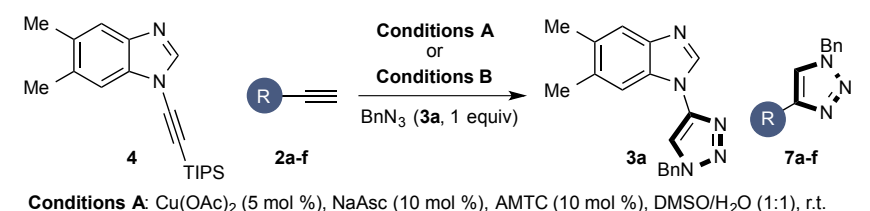

Conditions A: $\mathrm{Cu}(\mathrm{OAC})_{2}(5 \mathrm{~mol} \%)$, NaAsc (10 mol \%), AMTC (10 mol \%), DMSO/H $\mathrm{H}_{2} \mathrm{O}$ (1:1), r.t. Conditions B: $\mathrm{Cu}(\mathrm{OAc})_{2}(5 \mathrm{~mol} \%), \operatorname{TBAF}(1.1$ equiv), MeCN, r.t.

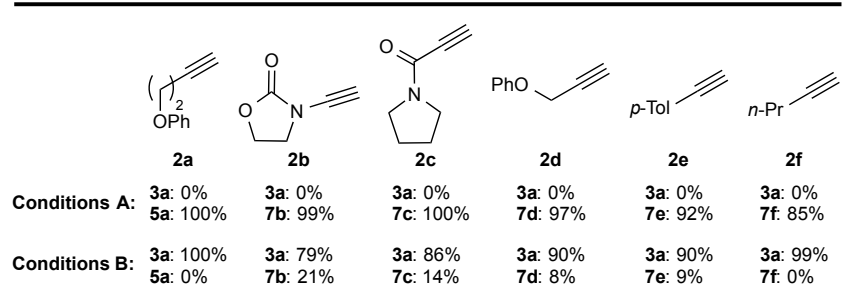

Scheme 4. Competition reactions of 4 with a range of alkynes 2a-f using conditions A and B. Isolated yields. 
Activated alkynes $\mathbf{2 b}$ and $\mathbf{2 c}$ display enhanced reactivity in CuAAC reactions over propargyl substrates (e.g., 2d) as well as aromatic (2e) and aliphatic alkynes (2f). ${ }^{9}$ Under alkyne-selective conditions (Conditions A), full conversion to the expected triazoles 7a-f was observed, with isolated yields $>85 \%$. When changing to the ynamine-selective conditions (Conditions B), chemoselectivity remained in favor of the ynamine, although the level of selectivity was lower for more reactive alkynes (2b, 2c) as could be anticipated based on previous studies. ${ }^{14}$

Orthogonal control was further explored first by use of the diyne 8 (Scheme 5). Preparation of the regioisomeric bis-triazole products $\mathbf{1 1 a}$ and $\mathbf{1 1} \mathbf{b}$ could be achieved either by reacting at the terminal alkyne then the ynamine (Conditions A then B) or by reaction at the ynamine followed by the terminal alkyne (Conditions B then A). This was not conditional on strategic use of a particular azide: The overall yield of 11a and 11b using both approaches was $>90 \%$, further demonstrating the orthogonality of the approach as well as establishing a useful method for sequential ligation in a multifunctional system.

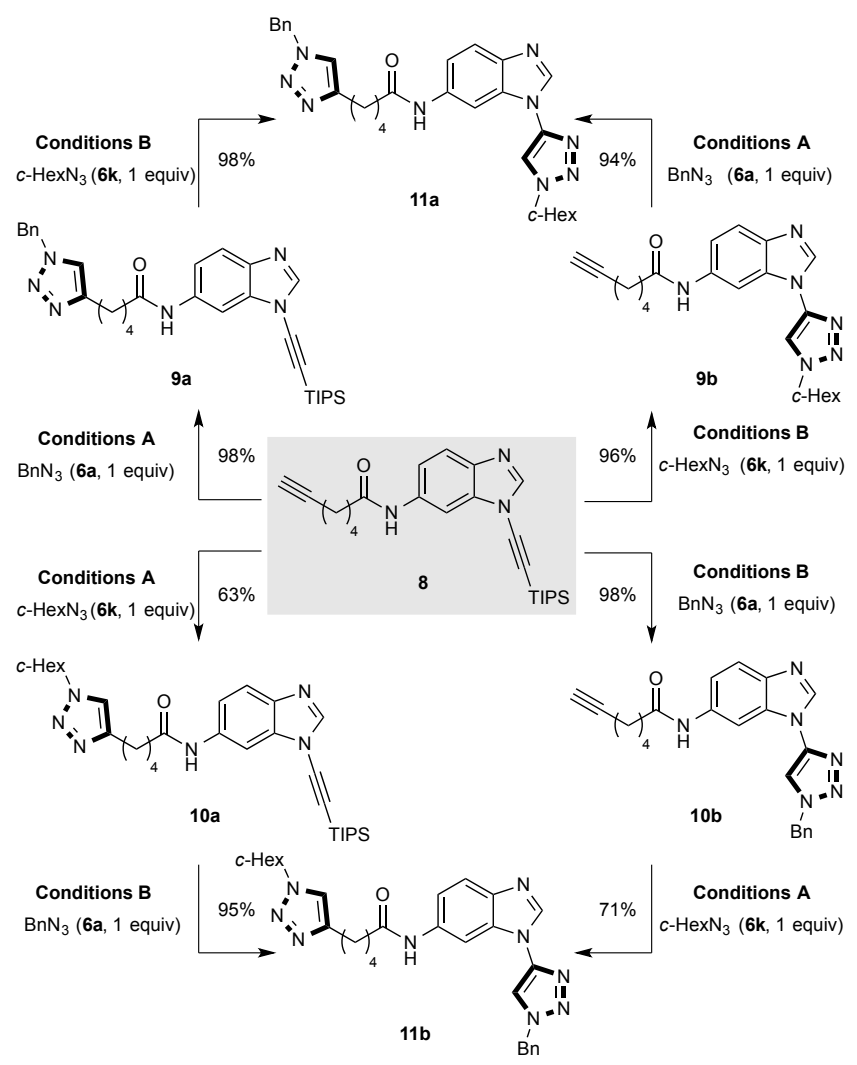


Scheme 5. Orthogonal CuAAC reactions on a diyne scaffold. 8. Isolated yields.

Lastly, we investigated the ability to simultaneously control both chemoselectivity at the alkyne as well as the azide component through exploitation of chelate-assistance to afford control over the azide (Scheme 6). ${ }^{7}$ Under alkyne-selective conditions (Conditions A), the multicomponent reaction of $\mathbf{2 e}, \mathbf{4}$, $\mathbf{6 a}$, and $\mathbf{6} \mathbf{f}$ delivers product pair $\mathbf{3 a}$ and $\mathbf{1 2}$ via first $\mathrm{CuAAC}$ reaction of $\mathbf{2 e}$ with the more reactive azide 6f. ${ }^{7 \mathrm{a}}$ Addition of TBAF liberates the reactive ynamine allowing the second CuAAC reaction with azide 6a to take place. Alternatively, the orthogonal product pair, $\mathbf{3 f}$ and $\mathbf{7 e}$, is generated using the ynamine-selective protocol (Conditions B) to promote the reaction of ynamine with $\mathbf{6 f}$ and the alkyne with 6a.

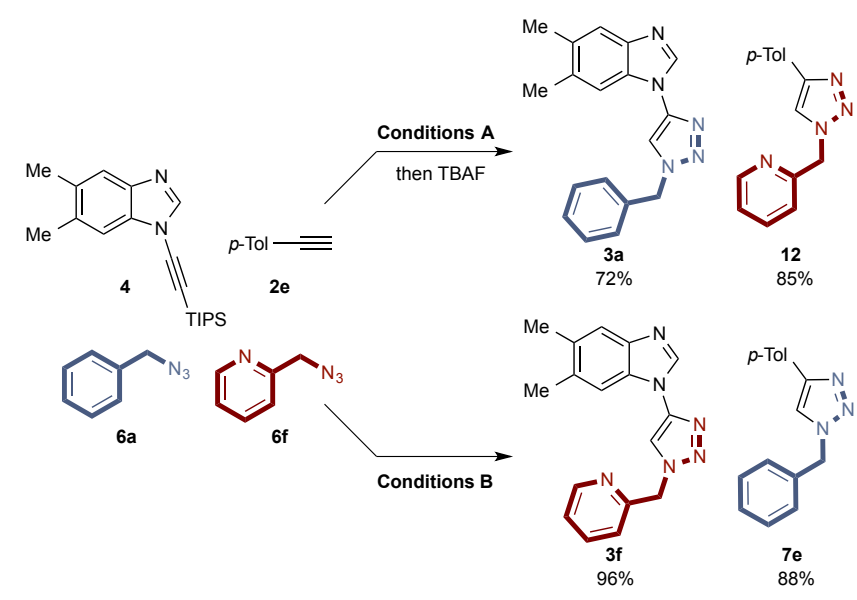

Scheme 6. Reactivity pairing of sequential $\mathrm{CuAAC}$ reactions. Isolated yields.

\section{CONCLUSIONS}

In summary, we have shown that orthogonal control of alkyne reactivity in CuAAC reactions can be controlled by the strategic use of a silyl protecting group. This allows the development of the first conditional orthogonal strategy for alkynes in CuAAC reactions, and displays excellent chemoselectivity profiles in single competition experiments as well as within multi-component reactions. We envisage the flexibility of this approach will provide new opportunities for chemoselective $\mathrm{CuAAC}$ reactions in a number of research areas, from chemical biology to materials science.

\section{EXPERIMENTAL SECTION}


General. All reagents and solvents were obtained from commercial suppliers and were used without further purification unless otherwise stated. Purification was carried out according to standard laboratory methods. Intermediates $4,{ }^{16} \mathbf{2 a},{ }^{17,18} \mathbf{6 b},{ }^{19} \mathbf{6 d},{ }^{20} \mathbf{6 e},{ }^{21} \mathbf{6 g},{ }^{22} \mathbf{6 h},{ }^{23} \mathbf{6 i},{ }^{24} \mathbf{6 j},{ }^{25} \mathbf{2 b},{ }^{16} \mathbf{2 c},{ }^{26} \mathbf{2 d},{ }^{27} \mathbf{8},{ }^{15}$ and $\mathrm{AMTC}^{28}$ were prepared according to literature procedures. Dry solvents for reactions were either obtained from a PureSolv SPS-400-5 solvent purification system, transferred to and stored in a septum-sealed oven-dried flask over previously activated $4 \AA$ molecular sieves and purged with and stored under nitrogen, or either used as obtained from suppliers without further purification. Reactions were carried out using conventional glassware (preparation of intermediates). Purging refers to a vacuum/nitrogen-refilling procedure. Room temperature was generally $18{ }^{\circ} \mathrm{C}$. Reactions were carried out at elevated temperatures using a temperature-regulated hotplate/stirrer.

Purification. Thin layer chromatography was carried out using Merck silica plates coated with fluorescent indicator UV254. These were analyzed under $254 \mathrm{~nm}$ UV light or developed using potassium permanganate solution. Normal phase flash chromatography was carried out using ZEOprep 60 HYD 40-63 $\mu \mathrm{m}$ silica gel.

Analysis. Fourier Transformed Infra-Red (FTIR) spectra were obtained on a Shimadzu IRAffinity-1 machine. $1 \mathrm{H}$ and 13C NMR spectra were obtained on either a Bruker AV 400 at $400 \mathrm{MHz}$ and 100 MHz, respectively, or Bruker DRX 500 at $500 \mathrm{MHz}$ and $126 \mathrm{MHz}$, respectively. Chemical shifts are reported in ppm and coupling constants are reported in $\mathrm{Hz}$ with $\mathrm{CDCl}_{3}$ referenced at $7.26 \mathrm{ppm}(1 \mathrm{H})$ and $77.0 \mathrm{ppm}\left({ }^{13} \mathrm{C}\right)$, DMSO-d6 referenced at $2.50 \mathrm{ppm}\left({ }^{1} \mathrm{H}\right)$ and $39.5 \mathrm{ppm}\left({ }^{13} \mathrm{C}\right)$, and MeOD referenced at $4.78 \mathrm{ppm}\left({ }^{1} \mathrm{H}\right)$ and $49.2 \mathrm{ppm}\left({ }^{13} \mathrm{C}\right)$. High resolution mass spectroscopy was recorded on a Bruker maXis Impact TOF mass spectrometer, equipped with an ESI interface, over a mass range of $50-1000 \mathrm{Da}$, with a scan time of $1 \mathrm{~s}$. Reverse phase HPLC data was obtained on an Agilent 1200 series HPLC using a Machery-Nagel Nucleodur C18 column. Analysis was performed using a gradient method, eluting with $5-80 \% \mathrm{MeCN} / \mathrm{H}_{2} \mathrm{O}$ over 50 minutes at a flow rate of $1 \mathrm{~mL} / \mathrm{min}$. Samples for HPLC analysis were prepared through the removal of $30 \mu \mathrm{L}$ of the reaction mixture in which $100 \mu \mathrm{L}$ of EDTA solution $(10 \mathrm{mg} / \mathrm{mL})$ was added. The product was extracted with $100 \mu \mathrm{L}$ of DCM. The organics were concentrated, and the product was dissolved in $\mathrm{MeOH}(1 \mathrm{~mL})$. To $110 \mu \mathrm{L}$ of this solution was added $5 \mu \mathrm{L}$ of 2-bromopyrimidine solution $(10 \mathrm{mg} / \mathrm{mL}$ in $\mathrm{MeOH})$ for $\mathrm{HPLC}$ 
analysis. Note: Due to the high heteroatom count in specific products, ${ }^{13} \mathrm{C} N M R$ analysis was not possible due to issues with relaxation.

General Procedures. General Procedure A for the formation of 3a-j (Scheme 3). To a solution of 5,6dimethyl-1-((triisopropylsilyl)ethynyl)-1H-benzo[d]imidazole (45 mg, $0.14 \mathrm{mmol}, 1$ equiv), (but-3-yn1-yloxy)benzene (20 mg, $0.14 \mathrm{mmol}, 1$ equiv) and azide 6a-j ( $0.14 \mathrm{mmol}, 1$ equiv) at $\mathrm{rt}$ in $\mathrm{MeCN}$ (1 $\mathrm{mL}$ ) was added TBAF ( $49 \mu \mathrm{L}, 0.15 \mathrm{mmol}, 1.1$ equiv) followed by $\mathrm{Cu}(\mathrm{OAc})_{2}(1 \mathrm{mg}, 0.007 \mathrm{mmol}, 0.05$ equiv). The reaction was stirred for $16 \mathrm{~h}$, after which EtOAc $(10 \mathrm{~mL})$ was added. The mixture was washed with EDTA $(10 \mathrm{mg} / \mathrm{mL}, 10 \mathrm{~mL})$ and brine $(2 \times 10 \mathrm{~mL})$, dried over $\mathrm{Na}_{2} \mathrm{SO}_{4}$, and concentrated under vacuum. The resulting residue was purified by flash chromatography (silica gel) to provide the desired compound.

General Procedure B for the formation of 5a-j (Scheme 3). To a solution of 5,6-dimethyl-1((triisopropylsilyl)ethynyl)-1H-benzo[d]imidazole (45 mg, $0.14 \mathrm{mmol}, 1$ equiv), (but-3-yn-1yloxy)benzene (20 mg, $0.14 \mathrm{mmol}, 1$ equiv) and azide 5a-j (0.14 mmol, 1 equiv) at $\mathrm{rt}$ in $\mathrm{DMSO} / \mathrm{H}_{2} \mathrm{O}$ $(1 / 1,1 \mathrm{~mL})$ was added AMTC (3 mg, $0.014 \mathrm{mmol}, 0.1$ equiv) followed by $\mathrm{Cu}(\mathrm{OAc})_{2}(1 \mathrm{mg}, 0.007$ mmol, 0.05 equiv) and NaAsc ( $3 \mathrm{mg}, 0.014 \mathrm{mmol}, 0.1$ equiv). The reaction was stirred for $16 \mathrm{~h}$, after which EtOAc $(10 \mathrm{~mL})$ was added. The mixture was washed with EDTA $(10 \mathrm{mg} / \mathrm{mL}, 10 \mathrm{~mL})$ and brine ( $2 \times 10 \mathrm{~mL}$ ), dried over $\mathrm{Na}_{2} \mathrm{SO}_{4}$, and concentrated under vacuum. The resulting residue was purified by flash chromatography (silica gel) to provide the desired compound.

General Procedure $\mathrm{C}$ for the formation of 7a-f (Scheme 4). To a solution of 5,6-dimethyl-1((triisopropylsilyl)ethynyl)-1H-benzo[d]imidazole (132 mg, $0.41 \mathrm{mmol}, 1$ equiv), alkyne 2a-f $(0.41$ mmol, 1 equiv) and benzyl azide (53 $\mu \mathrm{L}, 0.41 \mathrm{mmol}, 1$ equiv) at $\mathrm{rt}$ in $\mathrm{DMSO} / \mathrm{H}_{2} \mathrm{O}(1 / 1,1 \mathrm{~mL})$ was added AMTC ( $9 \mathrm{mg}, 0.041 \mathrm{mmol}, 0.1$ equiv) followed by $\mathrm{Cu}(\mathrm{OAc})_{2}(3 \mathrm{mg}, 0.02 \mathrm{mmol}, 0.05$ equiv) and NaAsc (9 mg, $0.041 \mathrm{mmol}, 0.1$ equiv). The reaction was stirred for $16 \mathrm{~h}$, after which EtOAc was added. The mixture was washed with EDTA $(10 \mathrm{mg} / \mathrm{mL}, 10 \mathrm{~mL})$ and brine $(2 \times 10 \mathrm{~mL})$, dried over $\mathrm{Na}_{2} \mathrm{SO}_{4}$, and concentrated under vacuum. The resulting residue was purified by flash chromatography (silica gel) to provide the desired compound. 
1-(1-Benzyl-1H-1,2,3-triazol-4-yl)-5,6-dimethyl-1H-benzo[d]imidazole (3a). Prepared using General Procedure A. White solid (31 mg, $66 \%$ ). Purification on silica gel using hexane/EtOAc 3/7. ${ }^{1} \mathrm{H}$ NMR $\left(\mathrm{CDCl}_{3}, 400 \mathrm{MHz}\right): \delta 8.20$ (br. s, $\left.1 \mathrm{H}\right), 7.67$ (s, 1H), 7.58 (br. s, 1H), 7.39-7.45 (m, 4H), 7.37-7.34 (m, 2H), $5.62(\mathrm{~s}, 2 \mathrm{H}), 2.37$ (s, 6H). ${ }^{13} \mathrm{C}$ NMR $\left(\mathrm{CDCl}_{3}, 100 \mathrm{MHz}\right): \delta 143.2,142.4,140.4,134.0,133.6$, 132.2, 131.1 $129.5,129.3,128.3,120.7,113.5,111.3,55.2,20.7,20.3$. IR $v_{\max }$ (neat): 3086, 2922, $2854,1724,1584,1495,1459,1407,1284,1213,1053,867,718 \mathrm{~cm}^{-1}$. HRMS (ESI-TOF) $\mathrm{m} / z$ : [M + $\mathrm{H}]^{+}$Calcd for $\mathrm{C}_{18} \mathrm{H}_{18} \mathrm{~N}_{5}$ 304.1557; Found 304.1551.

5,6-Dimethyl-1-(1-octyl-1H-1,2,3-triazol-4-yl)-1H-benzo[d]imidazole (3b). Prepared using General Procedure A. White solid ( $88 \mathrm{mg}, 98 \%$ ). Purification on silica gel using hexane/EtOAc 3/7. ${ }^{1} \mathrm{H}$ NMR $\left(\mathrm{CDCl}_{3}, 500 \mathrm{MHz}\right): \delta 8.62$ (br. s, 1H), 7.77-7-68 (m, 3H), 4.45 (t, $\left.J=7.1 \mathrm{~Hz}, 2 \mathrm{H}\right), 2.40(\mathrm{~s}, 3 \mathrm{H}), 2.38$ (s, 3H), 2.00 (app. quint, $J=6.6 \mathrm{~Hz}, 2 \mathrm{H}), 1.40-1.26(\mathrm{~m}, 10 \mathrm{H}), 0.87(\mathrm{t}, J=7.2 \mathrm{~Hz}, 3 \mathrm{H}) .{ }^{13} \mathrm{C}$ NMR $\left(\mathrm{CDCl}_{3}, 100 \mathrm{MHz}\right): \delta 133.9,132.0,113.8,51.5,31.8,30.4,29.8,29.2,29.1,26.6,22.7,20.7,20.4$, 14.2. IR $v_{\max }$ (neat): $3135,2924,2857,1740,1597,1500,1470,1383,1292,1206,1156,1091,1053$, 1033, 949, 869, $785 \mathrm{~cm}^{-1}$. HRMS (ESI-TOF) $\mathrm{m} / z$ : $[\mathrm{M}+\mathrm{H}]^{+}$Calcd for $\mathrm{C}_{19} \mathrm{H}_{28} \mathrm{~N}_{5}$ 326.2339; Found 326.2323.

1-(1-((3s,5s,7s)-Adamantan-1-yl)-1H-1,2,3-triazol-4-yl)-5,6-dimethyl-1H-benzo[d]imidazole

$(3 c)$.

Prepared using General Procedure A. White solid (95 mg, 99\%). Purification on silica gel using hexane/EtOAc 3/7. ${ }^{1} \mathrm{H}$ NMR $\left(\mathrm{CDCl}_{3}, 400 \mathrm{MHz}\right): \delta 8.22$ (br. s, 1H), 7.82 (s, 1H), 7.60 (s, 1H), 7.47 (s, 1H), 2.39 (s, 3H), 2.38 (s, 3H), 2.33 (br. s, 9H), 1.83 (br. s, 6H). ${ }^{13} \mathrm{C} \mathrm{NMR}\left(\mathrm{CDCl}_{3}, 100 \mathrm{MHz}\right): \delta$ $142.4,142.1,140.7,133.5,132.1,131.4,120.7,111.4,110.6,61.0,43.1,35.9,29.8,29.6,20.7$, 20.4.IR $v_{\max }$ (neat): 3080, 2917, 2852, 1725, 1586, 1495, 1467, 1448, 1281, 1216, 1149, 1019, 948, 861, $844 \mathrm{~cm}^{-1}$. HRMS (ESI-TOF) $\mathrm{m} / z$ : $[\mathrm{M}+\mathrm{H}]^{+}$Calcd for $\mathrm{C}_{21} \mathrm{H}_{26} \mathrm{~N}_{5}$ 348.2183; Found 348.2179.

(S)-5,6-Dimethyl-1-(1-(1-phenylethyl)-1H-1,2,3-triazol-4-yl)-1H-benzo[d]imidazole (3d). Prepared using General Procedure A. White solid (46 mg, 95\%). Purification on silica gel using hexane/EtOAc 3/7. ${ }^{1} \mathrm{H} \mathrm{NMR}\left(\mathrm{CDCl}_{3}, 400 \mathrm{MHz}\right): \delta 8.20$ (br. s, $\left.1 \mathrm{H}\right), 7.63$ (s, $\left.1 \mathrm{H}\right), 7.59$ (br. s, $\left.1 \mathrm{H}\right), 7.45-7-35$ (m, 6H), 
$5.88(\mathrm{q}, J=7.0 \mathrm{~Hz}, 1 \mathrm{H}), 2.37(\mathrm{~s}, 6 \mathrm{H}), 2.09$ (d, $J=7.1 \mathrm{~Hz}, 3 \mathrm{H}) .{ }^{13} \mathrm{C} \mathrm{NMR}\left(\mathrm{CDCl}_{3}, 100 \mathrm{MHz}\right): \delta 139.3$, $133.6,132.3,129.4,126.7,120.7,112.6,111.5,61.5,29.8,21.3,20.7,20.4 .4$ signals not observed/coincident. IR $v_{\max }$ (neat): $3110,2926,2857,1724,1590,1498,1459,1383,1286,1212$, 1143, 910, $731 \mathrm{~cm}^{-1}$. HRMS (ESI-TOF) $m / z:[\mathrm{M}+\mathrm{H}]^{+}$Calcd for $\mathrm{C}_{19} \mathrm{H}_{20} \mathrm{~N}_{5}$ 318.1713; Found 318.1704 .

(S)-2-((tert-Butoxycarbonyl)amino)-6-(4-(5,6-dimethyl-1H-benzo[d] imidazol-1-yl)-1H-1,2,3-triazol-1yl)hexanoic acid (3e). Prepared using General Procedure A. Yellow solid (56 mg, 94\%). Purification on silica gel using DCM/MeOH 9/1 + 0.01\% AcOH. ${ }^{1} \mathrm{H}$ NMR $\left(\mathrm{CDCl}_{3}, 400 \mathrm{MHz}\right): \delta 9.80$ (br. s, $\left.1 \mathrm{H}\right)$, 8.79 (s, 1H), 7.91 (s, 1H), 7.71 (s, 1H), 4.60 (br. s, 2H), 4.08 (br. s, 1H), 2.48 (s, 6H), 2.08 (br. s, 2H), 1.89 (br. s, $1 \mathrm{H}), 1.74$ (br. s, $1 \mathrm{H}), 1.51$ (br. s, $2 \mathrm{H}), 1.41$ (s, 9H). ${ }^{13} \mathrm{C} \mathrm{NMR}\left(\mathrm{CDCl}_{3}, 100 \mathrm{MHz}\right.$ ): $\delta$ 175.9, $158.1,141.0,139.2,138.9,119.9,116.0,114.8,80.4,54.6,52.4,32.2,30.6,28.7,23.9,20.7,20.5 .2$ signals not observed/coincident. IR $v_{\max }$ (neat): 3382, 3127, 2974, 2867, 2485, 2233, 2071, 1686, 1591, 1422, 1392, 1368, 1247, 1165, 1119, 1052, $974 \mathrm{~cm}^{-1}$. HRMS (ESI-TOF) $\mathrm{m} / z:[\mathrm{M}+\mathrm{H}]^{+}$Calcd for $\mathrm{C}_{22} \mathrm{H}_{31} \mathrm{~N}_{6} \mathrm{O}_{4} 443.2401$; Found 443.2389.

5,6-Dimethyl-1-(1-(pyridin-2-ylmethyl)-1H-1,2,3-triazol-4-yl)-1H-benzo[d]imidazole (3f). Prepared using General Procedure A. Yellow oil (83 mg, 99\%). Purification on silica gel using hexane/EtOAc 3/7. ${ }^{1} \mathrm{H}$ NMR $\left(\mathrm{CDCl}_{3}, 500 \mathrm{MHz}\right): \delta 8.61(\mathrm{~d}, J=4.5 \mathrm{~Hz}, 1 \mathrm{H}), 8.24(\mathrm{~s}, 1 \mathrm{H}), 8.03(\mathrm{~s}, 1 \mathrm{H}), 7.72(\mathrm{dt}, J=$ 7.7, 1.7 Hz, 1H), 7.57 (s, 1H), $7.45(\mathrm{~s}, 1 \mathrm{H}), 7.33$ (d, J=7.7 Hz, 1H), $7.29(\mathrm{~m}, 1 \mathrm{H}), 5.72(\mathrm{~s}, 2 \mathrm{H}), 2.36$ (s, 6H). ${ }^{13} \mathrm{C} \mathrm{NMR}\left(\mathrm{CDCl}_{3}, 100 \mathrm{MHz}\right): \delta 153.6,150.1,143.0,142.3,140.4,137.6,133.6,133.5,133.4$, $132.3,132.2,132.1,131.0,123.8,122.8,120.6,114.3,111.3$. IR $v_{\max }$ (neat): $3105,2960,2917,2852$, 1586, 1502, 1465, $1435 \mathrm{~cm}^{-1}$. HRMS (ESI-TOF) $\mathrm{m} / z$ : $[\mathrm{M}+\mathrm{H}]^{+}$Calcd for $\mathrm{C}_{17} \mathrm{H}_{17} \mathrm{~N}_{6}$ 305.1509; Found 305.1508 .

N-(2-(4-(5,6-Dimethyl-1H-benzo[d]imidazol-1-yl)-1H-1,2,3-triazol-1-yl)ethyl)-N-methyl-7nitrobenzo[c][1,2,5]oxadiazol-4-amine (3g). Prepared using General Procedure A. Red solid (119 mg, 95\%). Purification on silica gel using hexane/EtOAc 1/9. ${ }^{1} \mathrm{H}$ NMR $\left(\mathrm{CDCl}_{3}, 400 \mathrm{MHz}\right): \delta 8.48(\mathrm{~m}, 2 \mathrm{H})$, 7.63 (s, 1H), 7.49 (br. s, 1H), $6.38(\mathrm{~d}, J=9.3 \mathrm{~Hz}, 1 \mathrm{H}), 4.98$ (t, $J=4.5 \mathrm{~Hz}, 2 \mathrm{H}), 3.45-3.42(\mathrm{~m}, 2 \mathrm{H})$, 
$2.39(\mathrm{~d}, J=4.7 \mathrm{~Hz}, 6 \mathrm{H}), 2.03(\mathrm{~s}, 3 \mathrm{H}) .1$ signal not observed/coincident. IR $v_{\max }$ (neat): 3599, 3108, $3082,2917,2852,1613,1597,1550,1424,1288,1216,1149,1087,1002,918,732 \mathrm{~cm}^{-1}$. HRMS (ESI-TOF) $m / z$ : $[\mathrm{M}+\mathrm{H}]^{+}$Calcd for $\mathrm{C}_{20} \mathrm{H}_{20} \mathrm{~N}_{9} \mathrm{O}_{3}$ 434.1684; Found 434.1613.

N-(3-(4-(5,6-Dimethyl-1H-benzo[d]imidazol-1-yl)-1H-1,2,3-triazol-1-yl)propyl)-5-

(dimethylamino)naphthalene-1-sulfonamide (3h). Prepared using General Procedure A. Yellow solid (43 mg, 92\%). Purification on silica gel using DCM/MeOH 9/1. ${ }^{1} \mathrm{H}$ NMR $\left(\mathrm{CDCl}_{3}, 500 \mathrm{MHz}\right): \delta 8.52$ $(\mathrm{d}, J=8.6 \mathrm{~Hz}, 1 \mathrm{H}), 8.27(\mathrm{~d}, J=8.6 \mathrm{~Hz}, 1 \mathrm{H}), 8.24(\mathrm{~s}, 1 \mathrm{H}), 8.21(\mathrm{~d}, J=8.6 \mathrm{~Hz}, 1 \mathrm{H}), 7.80(\mathrm{~s}, 1 \mathrm{H}), 7.61$ (s, 1H), $7.55(\mathrm{t}, J=7.6 \mathrm{~Hz}, 1 \mathrm{H}), 7.50-7.46(\mathrm{~m}, 2 \mathrm{H}), 7.16(\mathrm{~d}, J=7.6 \mathrm{~Hz}, 1 \mathrm{H}), 5.38(\mathrm{t}, J=6.5 \mathrm{~Hz}, 1 \mathrm{H})$, $4.54(\mathrm{t}, J=5.5 \mathrm{~Hz}, 2 \mathrm{H}), 2.94(\mathrm{q}, J=6.2 \mathrm{~Hz}, 2 \mathrm{H}), 2.86(\mathrm{~s}, 6 \mathrm{H}), 2.39(\mathrm{~s}, 6 \mathrm{H}), 2.15(\mathrm{~m}, 2 \mathrm{H}) .{ }^{13} \mathrm{C}$ NMR $\left(\mathrm{CDCl}_{3}, 100 \mathrm{MHz}\right): \delta 152.4,142.6,142.4,140.5,134.2,133.8,132.4,131.0,130.1,129.9,129.6$, $128.9,123.4,120.7,118.3,115.5,114.7,111.4,47.7,45.5,39.9,30.2,20.7,20.4$. IR $v_{\max }$ (neat): 3127 , 2924, 2857, 2794, 1750, 1597, 1457, 1396, 1325, 1312, 1234, 1146, 1092, 1042, 951, $789 \mathrm{~cm}^{-1}$. HRMS (ESI-TOF) $m / z$ : $[\mathrm{M}+\mathrm{H}]^{+}$Calcd for $\mathrm{C}_{26} \mathrm{H}_{30} \mathrm{~N}_{7} \mathrm{O}_{2} \mathrm{~S}$ 504.2176; Found 504.2153.

1-(3',6'-Dihydroxy-3-oxo-3H-spiro[isobenzofuran-1,9'-xanthen]-5-yl)-3-(3-(4-(5,6-dimethyl-1Hbenzo[d]imidazol-1-yl)-1H-1,2,3-triazol-1-yl)propyl)thiourea (3i). Prepared using General Procedure A. Red solid (66 mg, 85\%). Purification on silica gel using DCM/MeOH 9/1). ${ }^{1} \mathrm{H}$ NMR $\left(\mathrm{CDCl}_{3}, 400\right.$ MHz): $\delta 9.32$ (br. s, 1H), 9.00 (s, 1H), 8.45 (s, 1H), 7.86 (d, J=8.0 Hz, 1H), 7.82 (s, 1H), 7.65-7.62 (m, 2H), $7.16(\mathrm{~d}, J=8.3 \mathrm{~Hz}, 1 \mathrm{H}), 6.72-6.68(\mathrm{~m}, 2 \mathrm{H}), 6.60-6.56(\mathrm{~m}, 3 \mathrm{H}), 4.66(\mathrm{t}, J=6.9 \mathrm{~Hz}, 2 \mathrm{H}), 3.51$ (s, 2H), 3.16 (s, 2H), 2.40 (s, 3H), 2.38 (s, 3H).IR $v_{\max }$ (neat): 2924, 2113, 1716, 1593, 1541, 1457, 1381, 1303, 1245, 1210, $854 \mathrm{~cm}^{-1}$. HRMS (ESI-TOF) $\mathrm{m} / z$ : $[\mathrm{M}-\mathrm{H}]^{-}$Calcd for $\mathrm{C}_{35} \mathrm{H}_{28} \mathrm{~N}_{7} \mathrm{O}_{5} \mathrm{~S} 658.1878$; Found $642.1632[\mathrm{M}-\mathrm{OH}]-{ }^{13} \mathrm{C}$ NMR could not be obtained due to relaxation issues.

N-(2-(2-(2-(2-(4-(5,6-Dimethyl-1H-benzo[d]imidazol-1-yl)-1H-1,2,3-triazol-1yl)ethoxy)ethoxy)ethoxy)ethyl)-5-((3aS,4S,6aR)-2-oxohexahydro-1H-thieno[3,4-d]imidazol-4yl)pentanamide (3j). Prepared using General Procedure A. White solid (24 mg, 87\%). Purification on silica gel using DCM/MeOH 9/1. ${ }^{1} \mathrm{H}$ NMR $\left(\mathrm{CDCl}_{3}, 400 \mathrm{MHz}\right): \delta 8.52$ (s, 2H), 7.63 (br. s, $\left.1 \mathrm{H}\right), 7.55$ 
(br. s, 1H), $4.71(\mathrm{t}, J=5.2 \mathrm{~Hz}, 2 \mathrm{H}), 4.45(\mathrm{dd}, J=7.8,4.9 \mathrm{~Hz}, 1 \mathrm{H}), 4.25(\mathrm{dd}, J=7.9,4.5 \mathrm{~Hz}, 1 \mathrm{H}), 3.99$ (t, $J=4.9 \mathrm{~Hz}, 2 \mathrm{H}), 3.69-3.66(\mathrm{~m}, 2 \mathrm{H}), 3.64-3.62(\mathrm{~m}, 2 \mathrm{H}), 3.58-3.55(\mathrm{~m}, 2 \mathrm{H}), 3.49-3.47(\mathrm{~m}, 2 \mathrm{H})$, $3.38(\mathrm{t}, J=5.4 \mathrm{~Hz}, 2 \mathrm{H}), 3.24(\mathrm{t}, J=5.4 \mathrm{~Hz}, 2 \mathrm{H}), 3.17-3.12(\mathrm{~m}, 1 \mathrm{H}), 2.88(\mathrm{dd}, J=12.8,5.0 \mathrm{~Hz}, 1 \mathrm{H})$, $2.68(\mathrm{~d}, J=12.7 \mathrm{~Hz}, 1 \mathrm{H}), 2.41(\mathrm{~s}, 3 \mathrm{H}), 2.40(\mathrm{~s}, 3 \mathrm{H}), 2.17-2.13(\mathrm{~m}, 2 \mathrm{H}), 1.73-1.50(\mathrm{~m}, 4 \mathrm{H}), 1.42-1.34$ (m, 2H). ${ }^{13} \mathrm{C} \mathrm{NMR}\left(\mathrm{CDCl}_{3}, 100 \mathrm{MHz}\right): \delta$ 176.0, 166.1, 135.2, 133.9, 120.6, 118.2, 112.9, 71.5, 71.4, $71.1,70.5,63.3,61.6,57.0,52.3,41.0,40.2,36.7,29.7,29.4,26.8,20.7,20.3$. IR $v_{\max }$ (neat): 3375 , 3124, 2922, 2867, 2470, 2068, 1690, 1645, 1591, 1454, 1098, $1055 \mathrm{~cm}^{-1}$. HRMS (ESI-TOF) $\mathrm{m} / \mathrm{z}:[\mathrm{M}+$ $\mathrm{H}]^{+}$Calcd for $\mathrm{C}_{28} \mathrm{H}_{43} \mathrm{~N}_{8} \mathrm{O}_{5} \mathrm{~S}$ 615.3072; Found 615.3149.

1-Benzyl-4-(2-phenoxyethyl)-1H-1,2,3-triazole (5a). Prepared using General Procedure B. White solid (81 mg, quant). Purification on silica gel using hexane/EtOAc 3/7. ${ }^{1} \mathrm{H} \mathrm{NMR}\left(\mathrm{CDCl}_{3}, 400 \mathrm{MHz}\right)$ : $\delta$ 7.40-7.34 (m, 4H), 7.29-7.26 (m, 2H), 7.25-7.24 (m, 2H), 6.94 (tt, $J=7.4,1.1 \mathrm{~Hz}), 6.89-6.85$ (m, 2H), $5.50(\mathrm{~s}, 2 \mathrm{H}), 4.23(\mathrm{t}, J=6.5 \mathrm{~Hz}, 2 \mathrm{H}), 3.19$ (t, $J=6.5 \mathrm{~Hz}, 2 \mathrm{H}) .{ }^{13} \mathrm{C} \mathrm{NMR}\left(\mathrm{CDCl}_{3}, 100 \mathrm{MHz}\right): \delta$ $158.8,135.0,129.6,129.2,128.8,128.1,122.0,121.1,114.7,66.8,54.2,26.4$. IR $v_{\max }$ (neat): 3120 , $3071,3038,2958,2935,1604,1590,1496,1459,1251,1219,1176,1059,1042,890,828,789,752$, $720 \mathrm{~cm}^{-1}$. HRMS (ESI-TOF) $\mathrm{m} / \mathrm{z}$ : $[\mathrm{M}+\mathrm{Na}]^{+}$Calcd for $\mathrm{C}_{17} \mathrm{H}_{17} \mathrm{~N}_{3} \mathrm{ONa}$ 302.1264; Found 302.1256.

1-Octyl-4-(2-phenoxyethyl)-1H-1,2,3-triazole (5b). Prepared using General Procedure B. White solid (78 mg, 94\%). Purification on silica gel using hexane/EtOAc 3/7. ${ }^{1} \mathrm{H}$ NMR ( $\left.\mathrm{CDCl}_{3}, 400 \mathrm{MHz}\right): \delta 7.41$ (s, 1H), 7.29-7.25 (m, 2H), 6.96-6.89 (m, 3H), $4.30(\mathrm{t}, J=7.2 \mathrm{~Hz}, 2 \mathrm{H}), 4.24(\mathrm{t}, J=6.5 \mathrm{~Hz}, 2 \mathrm{H}), 3.21$ (t, $J=6.5 \mathrm{~Hz}, 2 \mathrm{H}), 1.87$ (app. quint, $J=7.1 \mathrm{~Hz}, 2 \mathrm{H}), 1.30-1.25(\mathrm{~m}, 10 \mathrm{H}), 0.87(\mathrm{t}, J=6.6 \mathrm{~Hz}, 3 \mathrm{H}) .{ }^{13} \mathrm{C}$ $\mathrm{NMR}\left(\mathrm{CDCl}_{3}, 100 \mathrm{MHz}\right): \delta$ 158.7, 144.6, 129.6, 121.7, 121.0, 114.6, 66.8, 50.3, 31.8, 30.4, 29.1, 29.0, 26.6, 26.3, 22.7, 14.1. IR $v_{\max }$ (neat): 3142, 2956, 2922, 2852, 1604, 1591, 1500, 1487, 1388, 1247, 1217, 1176, 1057, 1035, 886, 817, 756, $694 \mathrm{~cm}^{-1}$. HRMS (ESI-TOF) $\mathrm{m} / z:[\mathrm{M}+\mathrm{Na}]^{+}$Calcd for $\mathrm{C}_{18} \mathrm{H}_{27} \mathrm{~N}_{3} \mathrm{ONa}$ 324.2046; Found 324.2035.

1-((3s,5s,7s)-Adamantan-1-yl)-4-(2-phenoxyethyl)-1H-1,2,3-triazole (5c). Prepared using General Procedure B. White solid (81 mg, quant). Purification on silica gel using hexane/EtOAc $3 / 7 .{ }^{1} \mathrm{H}$ NMR 
$\left(\mathrm{CDCl}_{3}, 400 \mathrm{MHz}\right): \delta 7.49(\mathrm{~s}, 1 \mathrm{H}), 7.30-7.28(\mathrm{~m}, 2 \mathrm{H}), 7.00-6.90(\mathrm{~m}, 3 \mathrm{H}), 4.25(\mathrm{t}, J=6.6 \mathrm{~Hz}, 2 \mathrm{H})$

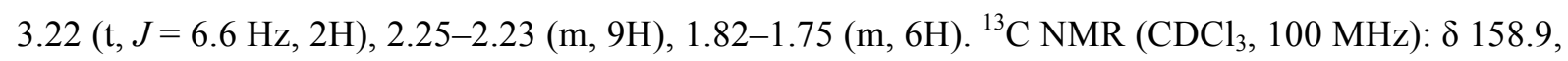
143.7, 129.6, 121.0, 118.2, 114.7, 67.1, 59.4, 43.1, 36.1, 29.6, 26.5. IR $v_{\max }$ (neat): 2909, 2857, 1599, $1590,1556,1500,1476,1457,1420,1348,1292,1243,1035,1016,780,759,694 \mathrm{~cm}^{-1}$. HRMS (ESITOF) $m / z:[\mathrm{M}+\mathrm{Na}]^{+}$Calcd for $\mathrm{C}_{20} \mathrm{H}_{25} \mathrm{~N}_{3} \mathrm{ONa} 346.1890$; Found 346.1881 .

(S)-4-(2-Phenoxyethyl)-1-(1-phenylethyl)-1H-1,2,3-triazole (5d). Prepared using General Procedure B. White solid (67 mg, 84\%). Purification on silica gel using hexane/EtOAc 3/7). ${ }^{1} \mathrm{H}$ NMR $\left(\mathrm{CDCl}_{3}, 400\right.$ MHz): $\delta 7.38-7.32(\mathrm{~m}, 8 \mathrm{H}), 6.94(\mathrm{tt}, J=7.4,1.1 \mathrm{~Hz}, 1 \mathrm{H}), 6.88-6.85(\mathrm{~m}, 2 \mathrm{H}), 5.78(\mathrm{q}, J=7.2 \mathrm{~Hz}, 1 \mathrm{H})$, $4.23(\mathrm{t}, J=6.5 \mathrm{~Hz}, 2 \mathrm{H}), 3.19(\mathrm{t}, J=6.5 \mathrm{~Hz}, 2 \mathrm{H}), 1.97(\mathrm{~d}, J=7.1 \mathrm{~Hz}, 3 \mathrm{H}) .{ }^{13} \mathrm{C} \mathrm{NMR}\left(\mathrm{CDCl}_{3}, 100\right.$ MHz): $\delta 158.7,144.6,140.2,129.6,129.1,128.5,126.6,121.0,120.8,114.7,66.8,60.2,25.4,21.4$. IR $v_{\max }$ (neat): $3170,3066,3030,3045,2941,2924,2870,1604,1588,1498,1463,1387,1366,1305$, $1251,1038,811,756,733,705,694 \mathrm{~cm}^{-1}$. HRMS (ESI-TOF) $\mathrm{m} / \mathrm{z}$ : $[\mathrm{M}+\mathrm{Na}]^{+}$Calcd for $\mathrm{C}_{18} \mathrm{H}_{19} \mathrm{~N}_{3} \mathrm{ONa}$ 316.1420; Found 316.1410.

(S)-2-((tert-Butoxycarbonyl)amino)-6-(4-(2-phenoxyethyl)-1H-1,2,3-triazol-1-yl)hexanoic acid (5e). Prepared using General Procedure B. White solid (46 mg, 82\%). Purification on silica gel using DCM/MeOH 9/1, 0.1\% AcOH. ${ }^{1} \mathrm{H}$ NMR (MeOD, $500 \mathrm{MHz}$ ): $\delta 7.83$ (br. s, $\left.1 \mathrm{H}\right), 7.24$ (t, $J=7.8 \mathrm{~Hz}$, 2H), 6.92-6.89 (m, 3H), $4.37(\mathrm{t}, J=7.1 \mathrm{~Hz}, 2 \mathrm{H}), 4.22(\mathrm{t}, J=6.5 \mathrm{~Hz}, 2 \mathrm{H}), 4.08-4.05(\mathrm{~m}, 1 \mathrm{H}), 3.15(\mathrm{t}, J$ $=6.4 \mathrm{~Hz}, 2 \mathrm{H}), 1.94-1.89(\mathrm{~m}, 2 \mathrm{H}), 1.87-1.80(\mathrm{~m}, 2 \mathrm{H}), 1.70-1.55(\mathrm{~m}, 2 \mathrm{H}), 1.42(\mathrm{~s}, 9 \mathrm{H}) .{ }^{13} \mathrm{C} \mathrm{NMR}$ (MeOD, $100 \mathrm{MHz}$ ): $\delta$ 176.0, 158.1, 130.5, 121.9, 115.6, 80.5, 67.7, 54.6, 52.2, 51.1, 32.3, 32.2, 29.4, 28.7, 26.9, 23.9.IR $v_{\max }$ (neat): 3398, 2952, 2922, 2868, 2482, 2242, 2071, 1688, 1600, 1368, 1167, 1119, $974 \mathrm{~cm}^{-1}$. HRMS (ESI-TOF) $m / z:[\mathrm{M}+\mathrm{Na}]^{+}$Calcd for $\mathrm{C}_{21} \mathrm{H}_{30} \mathrm{~N}_{4} \mathrm{O}_{5} \mathrm{Na}$ 441.2108; Found 441.2102 .

2-((4-(2-Phenoxyethyl)-1H-1,2,3-triazol-1-yl)methyl)pyridine (5f). Prepared using General Procedure B. White solid (60 mg, 79\%). Purification on silica gel using hexane/EtOAc 3/7. ${ }^{1} \mathrm{H} \mathrm{NMR}\left(\mathrm{CDCl}_{3}\right.$, $400 \mathrm{MHz}): \delta 8.60(\mathrm{~s}, 1 \mathrm{H}), 7.67(\mathrm{tt}, J=7.8,1.8 \mathrm{~Hz}, 1 \mathrm{H}), 7.60(\mathrm{~s}, 1 \mathrm{H}), 7.29-7.24(\mathrm{~m}, 4 \mathrm{H}), 7.15(\mathrm{~d}, J=$ 
$7.7 \mathrm{~Hz}, 1 \mathrm{H}), 6.93(\mathrm{tt}, J=7.3,1.2 \mathrm{~Hz}, 1 \mathrm{H}), 6.88(\mathrm{~m}, 1 \mathrm{H}), 5.63(\mathrm{~s}, 2 \mathrm{H}), 4.25(\mathrm{t}, J=6.5 \mathrm{~Hz}, 2 \mathrm{H}), 3.22(\mathrm{t}$, $J=6.5 \mathrm{~Hz}, 2 \mathrm{H}) .{ }^{13} \mathrm{C} \mathrm{NMR}\left(\mathrm{CDCl}_{3}, 100 \mathrm{MHz}\right): \delta 158.8,137.5,129.6,123.5,121.1,114.7,66.8,55.7$, 26.4. 3 signals not observed/coincident. IR $v_{\max }$ (neat): 3142, 2932, 1601, 1590, 1496, 1474, 1441, 1295, 1241, 1052, 1022, 1035, 998, 752, $692 \mathrm{~cm}^{-1}$. HRMS (ESI-TOF) $\mathrm{m} / z:[\mathrm{M}+\mathrm{Na}]^{+}$Calcd for $\mathrm{C}_{16} \mathrm{H}_{16} \mathrm{~N}_{4} \mathrm{ONa}$ 303.1216; Found 303.1214.

N-Methyl-7-nitro-N-(2-(4-(2-phenoxyethyl)-1H-1,2,3-triazol-1-yl)ethyl)benzo[c][1,2,5]oxadiazol-4amine (5g). Prepared using General Procedure B. Yellow solid (118 mg, 85\%). Purification on silica gel using hexane/EtOAc 1/9. ${ }^{1} \mathrm{H}$ NMR $\left(\mathrm{CDCl}_{3}, 500 \mathrm{MHz}\right): \delta 8.37$ (d, $\left.J=9.0 \mathrm{~Hz}, 1 \mathrm{H}\right), 7.86$ (br. s, $\left.1 \mathrm{H}\right)$, $7.24(\mathrm{t}, J=7.7 \mathrm{~Hz}, 2 \mathrm{H}), 6.92(\mathrm{t}, J=7.3 \mathrm{~Hz}, 1 \mathrm{H}), 6.84(\mathrm{~d}, J=8.0 \mathrm{~Hz}, 2 \mathrm{H}), 6.20(\mathrm{~d}, J=8.9 \mathrm{~Hz}, 1 \mathrm{H})$, $4.68(\mathrm{~m}, 2 \mathrm{H}), 4.01(\mathrm{t}, J=6.0 \mathrm{~Hz}, 2 \mathrm{H}), 3.03(\mathrm{t}, J=6.0 \mathrm{~Hz}, 2 \mathrm{H}), 1.34-1.29(\mathrm{~m}, 5 \mathrm{H}) . \mathrm{IR} v_{\max }$ (neat): $3142,1617,1556,1483,1429,1303,1281,1095,1035,1000,801,756,682 \mathrm{~cm}^{-1}$. HRMS (ESI-TOF) $m / z:[\mathrm{M}+\mathrm{Na}]^{+}$Calcd for $\mathrm{C}_{19} \mathrm{H}_{19} \mathrm{~N}_{7} \mathrm{O}_{4} \mathrm{Na}$ 432.1391; Found 432.1376. ${ }^{13} \mathrm{C}$ NMR could not be obtained due to relaxation issues.

5-(Dimethylamino)-N-(3-(4-(2-phenoxyethyl)-1H-1,2,3-triazol-1-yl)propyl)naphthalene-1-sulfonamide (5h). Prepared using General Procedure B. Yellow solid (38 mg, 87\%). Purification on silica gel using DCM/MeOH 9/1. ${ }^{1} \mathrm{H}$ NMR $\left(\mathrm{CDCl}_{3}, 400 \mathrm{MHz}\right): \delta 8.53(\mathrm{dt}, J=8.5,0.9 \mathrm{~Hz}, 1 \mathrm{H}), 8.27(\mathrm{dt}, J=8.5,0.9$ $\mathrm{Hz}, 1 \mathrm{H}), 8.18(\mathrm{dd}, J=7.3,1.2 \mathrm{~Hz}, 1 \mathrm{H}), 7.55(\mathrm{dd}, J=8.6,7.6 \mathrm{~Hz}, 1 \mathrm{H}), 7.47(\mathrm{dd}, J=8.5,7.2 \mathrm{~Hz}, 1 \mathrm{H})$, $7.33(\mathrm{~s}, 1 \mathrm{H}), 7.28-7.24(\mathrm{~m}, 2 \mathrm{H}), 7.16(\mathrm{~d}, J=7.6 \mathrm{~Hz}, 1 \mathrm{H}), 6.95-6.88(\mathrm{~m}, 3 \mathrm{H}), 5.41(\mathrm{t}, J=6.5 \mathrm{~Hz}, 1 \mathrm{H})$, $4.32(\mathrm{t}, J=6.6 \mathrm{~Hz}, 2 \mathrm{H}), 4.20(\mathrm{t}, J=6.3 \mathrm{~Hz}, 2 \mathrm{H}), 3.15(\mathrm{t}, J=6.3 \mathrm{~Hz}, 2 \mathrm{H}), 2.87(\mathrm{~s}, 6 \mathrm{H}), 2.01(\mathrm{~m}$, 4H). ${ }^{13} \mathrm{C}$ NMR $\left(\mathrm{CDCl}_{3}, 100 \mathrm{MHz}\right): \delta 158.7,152.2,134.5,130.8,130.0,129.8,129.6,128.7,123.3$, $121.0,118.6,115.4,114.7,66.7,46.9,45.5,40.1,32.0,30.4,29.8,26.2,14.3$. IR $v_{\max }$ (neat): 3293 , $3142,2935,2870,2790,1603,1590,1577,1500,1457,1409,1396,1314,1236,1145,1038,947$, 910, 791, 757, 731, $694 \mathrm{~cm}^{-1}$. HRMS (ESI-TOF) $\mathrm{m} / \mathrm{z}$ : $[\mathrm{M}+\mathrm{Na}]^{+}$Calcd for $\mathrm{C}_{25} \mathrm{H}_{29} \mathrm{~N}_{5} \mathrm{O}_{3} \mathrm{SNa} 502.1883$; Found 502.1862. 
1-(3',6'-Dihydroxy-3-oxo-3H-spiro[isobenzofuran-1,9'-xanthen]-5-yl)-3-(3-(4-(2-phenoxyethyl)-1H1,2,3-triazol-1-yl)propyl)thiourea (5i). Prepared using General Procedure B. Red solid (68 mg, 91\%). Purification on silica gel using DCM/MeOH 9/1. ${ }^{1} \mathrm{H}$ NMR $\left(\mathrm{CDCl}_{3}, 500 \mathrm{MHz}\right): \delta 8.72$ (br. s, $\left.1 \mathrm{H}\right), 8.38$ (s, 1H), $8.06(\mathrm{~s}, 1 \mathrm{H}), 7.83(\mathrm{~d}, J=7.7 \mathrm{~Hz}, 1 \mathrm{H}), 7.27(\mathrm{t}, J=7.1 \mathrm{~Hz}, 2 \mathrm{H}), 7.17(\mathrm{~d}, J=7.1 \mathrm{~Hz}, 1 \mathrm{H}), 6.95$ $(\mathrm{m}, 2 \mathrm{H}), 6.71(\mathrm{~s}, 3 \mathrm{H}), 6.58(\mathrm{q}, J=8.2 \mathrm{~Hz}, 3 \mathrm{H}), 4.45(\mathrm{t}, J=6.4 \mathrm{~Hz}, 2 \mathrm{H}), 4.22(\mathrm{t}, J=6.6 \mathrm{~Hz}, 4 \mathrm{H}), 3.51$ (m, 2H), 3.09 (t, $J=6.4 \mathrm{~Hz}, 2 \mathrm{H})$. IR $v_{\max }$ (neat): 2928, 1638, 1591, 1541, 1457, 1381, 1295, 1238, 1210, 1176, 1120, $852 \mathrm{~cm}^{-1}$. HRMS (ESI-TOF) $\mathrm{m} / \mathrm{z}$ : [M - H] Calcd for $\mathrm{C}_{34} \mathrm{H}_{28} \mathrm{~N}_{5} \mathrm{O}_{6} \mathrm{~S}$ 634.1766, found $618.2001[\mathrm{M}-\mathrm{OH}] .{ }^{13} \mathrm{C}$ NMR could not be obtained due to relaxation issues.

5-((3aS,4S,6aR)-2-Oxohexahydro-1H-thieno[3,4-d]imidazol-4-yl)-N-(2-(2-(2-(2-(4-(2-phenoxyethyl)1H-1,2,3-triazol-1-yl)ethoxy)ethoxy)ethoxy)ethyl)pentanamide (5j). Prepared using General Procedure B. White solid (32 mg, 88\%). Purification on silica gel using DCM/MeOH 9/1. ${ }^{1} \mathrm{H}$ NMR $\left(\mathrm{CDCl}_{3}, 400\right.$ MHz,): $\delta 7.96$ (br. s, 1H), 7.25 (t, $J=7.7 \mathrm{~Hz}, 2 \mathrm{H}), 6.92(\mathrm{~d}, J=7.2 \mathrm{~Hz}, 3 \mathrm{H}), 4.56(\mathrm{t}, J=4.6 \mathrm{~Hz}, 2 \mathrm{H})$, $4.47(\mathrm{dd}, J=7.6,5.6 \mathrm{~Hz}, 1 \mathrm{H}), 4.28(\mathrm{dd}, J=7.9,4.3 \mathrm{~Hz}, 1 \mathrm{H}), 4.24(\mathrm{t}, J=5.1 \mathrm{~Hz}, 2 \mathrm{H}), 3.88(\mathrm{t}, J=4.7$ $\mathrm{Hz}, 2 \mathrm{H}), 3.63-3.54(\mathrm{~m}, 8 \mathrm{H}), 3.50(\mathrm{t}, J=5.1 \mathrm{~Hz}, 2 \mathrm{H}), 3.37-3.33(\mathrm{~m}, 2 \mathrm{H}), 3.20-3.17(\mathrm{~m}, 3 \mathrm{H}), 2.90$ (dd, $J=12.5,4.8 \mathrm{~Hz}, 1 \mathrm{H}), 2.70(\mathrm{~d}, J=12.7 \mathrm{~Hz}, 1 \mathrm{H}), 2.19(\mathrm{t}, J=7.4 \mathrm{~Hz}, 2 \mathrm{H}), 1.75-1.54(\mathrm{~m}, 4 \mathrm{H}), 1.41$ (app. quint, $J=7.5 \mathrm{~Hz}, 2 \mathrm{H}) .{ }^{13} \mathrm{C} \mathrm{NMR}\left(\mathrm{CDCl}_{3}, 100 \mathrm{MHz}\right): \delta 176.1,166.1,160.2,130.5,121.9,115.7$, $71.6,71.5,71.4,71.2,10.6,70.4,67.8,63.4,61.6,57.0,51.5,41.1,40.3,36.7,29.7,29.5,27.0,26.8 .1$ signal not observed/coincident. IR $v_{\max }$ (neat): 3274, 3071, 2917, 2861, 1695, 1679, 1643, 1545, 1474, 1238, 1104, 1035, $756 \mathrm{~cm}^{-1}$. HRMS (ESI-TOF) $\mathrm{m} / z$ : $[\mathrm{M}+\mathrm{H}]^{+}$Calcd for $\mathrm{C}_{28} \mathrm{H}_{43} \mathrm{~N}_{6} \mathrm{O}_{6} \mathrm{~S} 591.2959$, found 591.2811 .

3-(1-Benzyl-1H-1,2,3-triazol-4-yl)oxazolidin-2-one (7b) ${ }^{29}$ Prepared using General Procedure C. White solid (99 mg, 99\%). Purification on silica gel using hexane/EtOAc 7/3. ${ }^{1} \mathrm{H}$ NMR $\left(\mathrm{CDCl}_{3}, 400 \mathrm{MHz}\right): \delta$ $7.82(\mathrm{~s}, 1 \mathrm{H}), 7.36-7.33(\mathrm{~m}, 3 \mathrm{H}), 7.28-7.26(\mathrm{~m}, 2 \mathrm{H}), 5.48(\mathrm{~s}, 2 \mathrm{H}), 4.56-4.52(\mathrm{~m}, 2 \mathrm{H}), 4.25-4.21(\mathrm{~m}$, 2H). ${ }^{13} \mathrm{C} \mathrm{NMR}\left(\mathrm{CDCl}_{3}, 100 \mathrm{MHz}\right): \delta 154.9,144.1,134.4,129.2,129.0,128.2,111.9,63.4,54.9,43.7$. 
(1-Benzyl-1H-1,2,3-triazol-4-yl)(pyrrolidin-1-yl)methanone (7c). Prepared using General Procedure C.

White solid (105 mg, quant). Purification on silica gel using hexane/EtOAc 7/3. ${ }^{1} \mathrm{H} \mathrm{NMR}\left(\mathrm{CDCl}_{3}, 400\right.$ MHz): $\delta 8.02(\mathrm{~s}, 1 \mathrm{H}), 7.36-7.34(\mathrm{~m}, 3 \mathrm{H}), 7.28-7.26(\mathrm{~m}, 2 \mathrm{H}), 5.52(\mathrm{~s}, 2 \mathrm{H}), 4.09(\mathrm{t}, J=6.7 \mathrm{~Hz}, 2 \mathrm{H})$, $3.62(\mathrm{t}, J=6.9 \mathrm{~Hz}, 2 \mathrm{H}), 1.97$ (app. quint, $J=7.1 \mathrm{~Hz}, 2 \mathrm{H}$ ), 1.88 (app. quint, $J=7.1 \mathrm{~Hz}, 2 \mathrm{H}$ ). ${ }^{13} \mathrm{C} \mathrm{NMR}$ $\left(\mathrm{CDCl}_{3}, 100 \mathrm{MHz}\right): \delta 159.5,145.5,134.1,129.3,129.0,128.3,127.6,54.3,48.7,47.0,26.6,23.8 . \mathrm{IR}$ $v_{\max }$ (neat): $3298,3099,2939,2861,2190,1600,1543,1498,1424,1342,1279,1229,1048 \mathrm{~cm}^{-1}$. HRMS (ESI-TOF) $m / z$ : [M + Na] $]^{+}$Calcd for $\mathrm{C}_{14} \mathrm{H}_{16} \mathrm{~N}_{4} \mathrm{ONa} 279.1216$; Found 279.1206.

1-Benzyl-4-(phenoxymethyl)-1H-1,2,3-triazole $(\mathbf{7 d}) .{ }^{30}$ Prepared using General Procedure C. White solid (106 mg, 97\%). Purification on silica gel using hexane/EtOAc 9/1. ${ }^{1} \mathrm{H}$ NMR $\left(\mathrm{CDCl}_{3}, 400 \mathrm{MHz}\right)$ : $\delta 7.52(\mathrm{~s}, 1 \mathrm{H}), 7.37-7.35(\mathrm{~m}, 3 \mathrm{H}), 7.29-7.25(\mathrm{~m}, 4 \mathrm{H}), 6.97-6.95(\mathrm{~m}, 3 \mathrm{H}), 5.52(\mathrm{~s}, 2 \mathrm{H}), 5.18(\mathrm{~s}, 2 \mathrm{H})$. ${ }^{13} \mathrm{C} \mathrm{NMR}\left(\mathrm{CDCl}_{3}, 100 \mathrm{MHz}\right): \delta 157.7,144.2,134.0,129.0,128.7,128.3,127.6,122.1,120.8,114.3$, $61.6,53.7$.

1-Benzyl-4-(p-tolyl)-1H-1,2,3-triazole (7e). ${ }^{31}$ Prepared using General Procedure C. White solid (94 $\mathrm{mg}, 92 \%)$. Purification on silica gel using hexane/EtOAc 9/1. ${ }^{1} \mathrm{H}$ NMR $\left(\mathrm{CDCl}_{3}, 400 \mathrm{MHz}\right): \delta 7.70$ (s, 1H), $7.68(\mathrm{~s}, 1 \mathrm{H}), 7.62(\mathrm{~s}, 1 \mathrm{H}), 7.39-7.36(\mathrm{~m}, 3 \mathrm{H}), 7.32-7.31(\mathrm{~m}, 1 \mathrm{H}), 7.30-7.29(\mathrm{~m}, 1 \mathrm{H}), 7.21(\mathrm{~s}$, 1H), $7.19(\mathrm{~s}, 1 \mathrm{H}), 5.56(\mathrm{~s}, 2 \mathrm{H}), 2.36(\mathrm{~s}, 3 \mathrm{H}) .{ }^{13} \mathrm{C} \mathrm{NMR}\left(\mathrm{CDCl}_{3}, 100 \mathrm{MHz}\right): \delta 148.4,138.1,134.9$, $129.6,129.3,128.9,128.2,127.9,125.7,119.3,54.3,21.4$.

1-Benzyl-4-propyl-1H-1,2,3-triazole (7f). ${ }^{32}$ Prepared using General Procedure C. White solid (70 mg, $85 \%)$. Purification on silica gel using hexane. ${ }^{1} \mathrm{H}$ NMR $\left(\mathrm{CDCl}_{3}, 400 \mathrm{MHz}\right): \delta 7.36-7.31(\mathrm{~m}, 3 \mathrm{H})$, 7.24-7.21 (m, 2H), $7.18(\mathrm{~s}, 1 \mathrm{H}), 5.47(\mathrm{~s}, 2 \mathrm{H}), 2.64(\mathrm{t}, J=7.4 \mathrm{~Hz}, 2 \mathrm{H}), 1.64($ sextet, $J=7.4 \mathrm{~Hz}, 2 \mathrm{H})$, $0.9(\mathrm{t}, J=7.4 \mathrm{~Hz}, 3 \mathrm{H}) .{ }^{13} \mathrm{C} \mathrm{NMR}\left(\mathrm{CDCl}_{3}, 100 \mathrm{MHz}\right): \delta 148.8,135.1,129.1,128.6,128.0,120.7,54.0$, $27.8,22.7,13.8$.

5-(1-Benzyl-1H-1,2,3-triazol-4-yl)-N-(1-((triisopropylsilyl)ethynyl)-1H-benzo[d] imidazol-6-

yl)pentanamide (9a). To a solution of $N$-(1-((triisopropylsilyl)ethynyl)-1H-benzo[d]imidazol-6- 
yl)hept-6-ynamide (50 mg, $0.119 \mathrm{mmol}, 1$ equiv.) in $\mathrm{MeOH} / \mathrm{H}_{2} \mathrm{O}(1 / 1,2 \mathrm{~mL})$ was added benzyl azide (15 $\mu \mathrm{L}, 0.119$ mmol, 1 equiv.), AMTC (3 mg, 0.012 mmol, 0.1 equiv.), $\mathrm{Cu}(\mathrm{OAc})_{2}$ ( $1 \mathrm{mg}, 0.006 \mathrm{mmol}$, 0.05 equiv.), and $\mathrm{NaAsc}(3 \mathrm{mg}, 0.012 \mathrm{mmol}, 0.1$ equiv.). The reaction was stirred at $\mathrm{rt}$ for $16 \mathrm{~h}$, after which DCM $(10 \mathrm{~mL})$ was added. The mixture was washed with aq. EDTA $(10 \mathrm{mg} / \mathrm{mL}, 10 \mathrm{~mL})$, brine ( $2 \times 10 \mathrm{~mL}$ ), dried over $\mathrm{Na}_{2} \mathrm{SO}_{4}$, and concentrated under reduced pressure. The resulting residue was purified by flash chromatography (silica gel, hexane/ EtOAc 3/7) to provide the desired product as a white solid (62 mg, 98\%). ${ }^{1} \mathrm{H} \mathrm{NMR}\left(\mathrm{CDCl}_{3}, 400 \mathrm{MHz}\right.$ ): $\delta 8.75$ (s, 1H), 8.24 (s, 1H), 8.05 (br. s, 1H), 7.64 (br. s, 1H), 7.35-7.30 (m, 4H), 7.23-7.21 (m, 3H), 5.45 (s, 2H), 2.71 (t, $J=6.9 \mathrm{~Hz}, 2 \mathrm{H}), 2.43$ (t, $J$ $=6.9 \mathrm{~Hz}, 2 \mathrm{H}), 1.79-1.70(\mathrm{~m}, 4 \mathrm{H}), 1.14(\mathrm{~s}, 21 \mathrm{H}) .{ }^{13} \mathrm{C} \mathrm{NMR}\left(\mathrm{CDCl}_{3}, 100 \mathrm{MHz}\right): \delta 171.8,136.3,134.8$, $129.2,128.8,128.1,120.6,117.0,102.6,73.5,54.2,37.1,29.8,28.6,25.1,25.0,18.7,11.3 .5$ signals not observed/coincident. IR $v_{\max }$ (neat): 3251, 2937, 2859, 2184, 1664, 1602, 1548, 1498, 1441, 1216, 909, 883, $730 \mathrm{~cm}^{-1}$. HRMS (ESI-TOF) $\mathrm{m} / z$ : $[\mathrm{M}+\mathrm{Na}]^{+}$Calcd for $\mathrm{C}_{32} \mathrm{H}_{42} \mathrm{~N}_{6} \mathrm{OSiNa}$ 577.3082; Found 577.3122.

N-(1-(1-Cyclohexyl-1H-1,2,3-triazol-4-yl)-1H-benzo[d]imidazol-6-yl)hept-6-ynamide (9b). To a solution of $N$-(1-((triisopropylsilyl)ethynyl)-1H-benzo[d]imidazol-6-yl)hept-6-ynamide (50 mg, 0.12 mmol, 1 equiv.) in MeCN ( $1 \mathrm{~mL}$ ) was added cyclohexylazide (15 mg, $0.12 \mathrm{mmol}, 1$ equiv.), TBAF (43 $\mu \mathrm{L}, 0.13 \mathrm{mmol}, 1.1$ equiv.), and $\mathrm{Cu}(\mathrm{OAc})_{2}(1 \mathrm{mg}, 0.006 \mathrm{mmol}, 0.05$ equiv.). The reaction was stirred at $\mathrm{rt}$ for $16 \mathrm{~h}$, after which DCM $(10 \mathrm{~mL})$ was added. The mixture was washed with aq. EDTA $(10 \mathrm{mg} / \mathrm{mL}, 10 \mathrm{~mL})$, brine $(2 \times 10 \mathrm{~mL})$, dried over $\mathrm{Na}_{2} \mathrm{SO}_{4}$, and concentrated under reduced pressure. The resulting residue was purified by flash chromatography (silica gel, 7/3 EtOAc/hexane) to provide the desired product as a white solid $(45 \mathrm{mg}, 96 \%) .{ }^{1} \mathrm{H} \mathrm{NMR}\left(\mathrm{CDCl}_{3}, 400 \mathrm{MHz}\right.$ ): $\delta 8.40$ (br. s, $\left.1 \mathrm{H}\right)$, 8.34 (s, 1H), 8.08 (br. s, 1H), 7.85 (s, 1H), 7.68 (br. s, 1H), 7.07 (d, $J=5.9 \mathrm{~Hz}, 1 \mathrm{H}), 4.48$ (tt, $J=11.8$, $3.6 \mathrm{~Hz}, 1 \mathrm{H}), 2.41(\mathrm{t}, J=7.4 \mathrm{~Hz}, 2 \mathrm{H}), 2.27(\mathrm{~d}, J=12.4 \mathrm{~Hz}, 2 \mathrm{H}), 2.21(\mathrm{dt}, J=7.2,2.1 \mathrm{~Hz}, 2 \mathrm{H}), 1.96(\mathrm{~m}$, $3 \mathrm{H}), 1.89-1.82(\mathrm{~m}, 4 \mathrm{H}), 1.59$ (app. quint, $J=7.8 \mathrm{~Hz}, 2 \mathrm{H}), 1.52-1.41(\mathrm{~m}, 2 \mathrm{H}), 1.32(\mathrm{dt}, J=12.8,2.7$ $\mathrm{Hz}, 2 \mathrm{H}) .{ }^{13} \mathrm{C} \mathrm{NMR}\left(\mathrm{CDCl}_{3}, 100 \mathrm{MHz}\right): \delta$ 171.5, 141.7, 135.1, 120.7, 116.1, 112.4, 102.7, 84.1, 68.9, 61.4, 37.2, 33.5, 28.0, 25.2, 25.1, 24.8, 18.3. 3 signals not observed/coincident. IR $v_{\max }$ (neat): 3289, 
$3259,3123,3080,2930,2855,1673,1604,1587,1550,1487,1442,1299,1240,911,803,730 \mathrm{~cm}^{-1}$. HRMS (ESI-TOF) $m / z$ : $[\mathrm{M}+\mathrm{Na}]^{+}$Calcd for $\mathrm{C}_{22} \mathrm{H}_{26} \mathrm{~N}_{6} \mathrm{ONa}$ 413.2060; Found 413.2049.

\section{5-(1-Cyclohexyl-1H-1,2,3-triazol-4-yl)-N-(1-((triisopropylsilyl)ethynyl)-1H-benzo[d]imidazol-6-}

yl)pentanamide (10a). To a solution of $N$-(1-((triisopropylsilyl)ethynyl)-1H-benzo[ $d]$ imidazol-6yl)hept-6-ynamide (50 mg, 0.119 mmol, 1 equiv.) in $\mathrm{MeOH} / \mathrm{H}_{2} \mathrm{O}$ (1/1, $2 \mathrm{~mL}$ ) was added cyclohexylazide (15 mg, $0.12 \mathrm{mmol}, 1$ equiv.), AMTC (3 mg, $0.012 \mathrm{mmol}, 0.1$ equiv.), $\mathrm{Cu}(\mathrm{OAc})_{2}(1$ mg, $0.006 \mathrm{mmol}, 0.05$ equiv.), and $\mathrm{NaAsc}(3 \mathrm{mg}, 0.012 \mathrm{mmol}, 0.1$ equiv.). The reaction was stirred at rt for $24 \mathrm{~h}$, after which DCM $(10 \mathrm{~mL})$ was added. The mixture was washed with aq. EDTA (10 $\mathrm{mg} / \mathrm{mL}, 10 \mathrm{~mL})$, brine $(2 \times 10 \mathrm{~mL})$, dried over $\mathrm{Na}_{2} \mathrm{SO}_{4}$, and concentrated under reduced pressure. The resulting residue was purified by flash chromatography (silica gel, hexane/ EtOAc 3/7) to provide the desired product as a white solid (42 mg, 63\%). ${ }^{1} \mathrm{H}$ NMR $\left(\mathrm{CDCl}_{3}, 400 \mathrm{MHz}\right.$,): $\delta 8.51$ (br. s, $\left.1 \mathrm{H}\right), 8.20$ $(\mathrm{d}, J=1.6 \mathrm{~Hz}, 1 \mathrm{H}), 8.01(\mathrm{~s}, 1 \mathrm{H}), 7.66(\mathrm{~d}, J=8.7 \mathrm{~Hz}, 1 \mathrm{H}), 7.32(\mathrm{dd}, J=8.6,1.7 \mathrm{~Hz}, 1 \mathrm{H}), 7.29(\mathrm{~s}, 1 \mathrm{H})$, $4.38(\mathrm{tt}, J=11.8,3.8 \mathrm{~Hz}, 1 \mathrm{H}), 2.75(\mathrm{t}, J=6.9 \mathrm{~Hz}, 2 \mathrm{H}), 2.45(\mathrm{t}, J=6.7 \mathrm{~Hz}, 2 \mathrm{H}), 2.18-2.15(\mathrm{~m}, 2 \mathrm{H})$, 1.92-1.87 (m, 2H), 1.83-1.65 (m, 6H), 1.48-1.37 (m, 2H), 1.27-1.21 (m, 2H), 1.16-1.14 (m, 21H).

${ }^{13} \mathrm{C} \mathrm{NMR}\left(\mathrm{CDCl}_{3}, 100 \mathrm{MHz}\right): \delta 171.8,147.2,143.8,138.2,136.2,135.0,120.8,118.7,117.0,102.6$, $90.1,73.4,60.1,37.2,33.7,28.6,25.3,25.3,25.1,25.0,18.8,11.3 .9$ signals not observed/coincident. IR $v_{\max }$ (neat): $3296,3132,3075,2935,2859,2184,1686,1664,1602,1547,1500,1483,1441,1376$, 1284, 1216, $1071 ?$ 998, 885, $680 \mathrm{~cm}^{-1}$. HRMS (ESI-TOF): m/z: $[\mathrm{M}+\mathrm{Na}]^{+}$Calcd for $\mathrm{C}_{31} \mathrm{H}_{46} \mathrm{~N}_{6} \mathrm{OSiNa}$ 569.3395; Found 569.3382.

N-(1-(1-Benzyl-1H-1,2,3-triazol-4-yl)-1H-benzo[d]imidazol-6-yl)hept-6-ynamide (10b). To a solution of $N$-(1-((triisopropylsilyl)ethynyl)-1H-benzo[d]imidazol-6-yl)hept-6-ynamide (50 mg, $0.12 \mathrm{mmol}, 1$ equiv.) in MeCN ( $1 \mathrm{~mL}$ ) was added benzyl azide (15 $\mu \mathrm{L}, 0.119$ mmol, 1 equiv.), TBAF (43 $\mu \mathrm{L}, 0.13$ mmol, 1.1 equiv.), and $\mathrm{Cu}(\mathrm{OAc})_{2}(1 \mathrm{mg}, 0.006 \mathrm{mmol}, 0.05$ equiv. $)$. The reaction was stirred at $\mathrm{rt}$ for 2 h, after which DCM $(10 \mathrm{~mL})$ was added. The mixture was washed with aq. EDTA $(10 \mathrm{mg} / \mathrm{mL}, 10$ $\mathrm{mL})$, brine $(2 \times 10 \mathrm{~mL})$, dried over $\mathrm{Na}_{2} \mathrm{SO}_{4}$, and concentrated under reduced pressure. The resulting residue was purified by flash chromatography (silica gel, 9/1 EtOAc/hexane) to provide the desired 
product as a white solid (47 mg, 98\%). ${ }^{1} \mathrm{H} \mathrm{NMR}\left(\mathrm{CDCl}_{3}, 400 \mathrm{MHz}\right.$,): $\delta 8.37$ (br. s, $\left.1 \mathrm{H}\right), 8.25$ (br. s, 1H), 8.01 (br. s, 1H), $7.88(\mathrm{~s}, 1 \mathrm{H}), 7.68(\mathrm{~d}, J=8.7 \mathrm{~Hz}, 1 \mathrm{H}), 7.39-7.34(\mathrm{~m}, 5 \mathrm{H}), 7.20(\mathrm{dd}, J=8.6,1.7$ $\mathrm{Hz}, 1 \mathrm{H}), 5.61(\mathrm{~s}, 2 \mathrm{H}), 3.12-3.08(\mathrm{~m}, 2 \mathrm{H}), 2.42(\mathrm{t}, J=7.3 \mathrm{~Hz}, 2 \mathrm{H}), 2.22(\mathrm{td}, J=7.2,2.7 \mathrm{~Hz}, 2 \mathrm{H}), 1.95$ $(\mathrm{t}, J=2.5 \mathrm{~Hz}, 1 \mathrm{H}), 1.88-1.80(\mathrm{~m} 2 \mathrm{H}) .{ }^{13} \mathrm{C} \mathrm{NMR}\left(\mathrm{CDCl}_{3}, 100 \mathrm{MHz}\right): \delta 171.3,135.2,134.1,129.4$, $129.2,128.3,120.6,116.2,114.2,102.5,84.2,68.8,55.2,37.1,28.0,24.7,19.7,18.3 .6$ signals not observed/coincident. IR $v_{\max }$ (neat): 2954, 2961, 2865, 1599, 1589, 1439, 1396, 1080, 1065, 1041, 866, $775 \mathrm{~cm}^{-1}$. HRMS (ESI-TOF): $\mathrm{m} / \mathrm{z}:[\mathrm{M}+\mathrm{Na}]^{+}$Calcd for $\mathrm{C}_{23} \mathrm{H}_{22} \mathrm{~N}_{6} \mathrm{ONa}$ 421.1747; Found 421.1732.

5-(1-Benzyl-1H-1,2,3-triazol-4-yl)-N-(1-(1-cyclohexyl-1H-1,2,3-triazol-4-yl)-1H-benzo[d]imidazol-6yl)pentanamide (11a). Method A: To a solution of 5-(1-benzyl-1H-1,2,3-triazol-4-yl)- $N-(1-$ ((triisopropylsilyl)ethynyl)-1H-benzo[d]imidazol-6-yl)pentanamide (64 mg, $0.12 \mathrm{mmol}, 1$ equiv.) in MeCN ( $1 \mathrm{~mL}$ ) was added cyclohexylazide (15 mg, $0.12 \mathrm{mmol}, 1$ equiv.), TBAF (43 $\mu \mathrm{L}, 0.13 \mathrm{mmol}$, 1.1 equiv.) and $\mathrm{Cu}(\mathrm{OAc})_{2}(1 \mathrm{mg}, 0.006 \mathrm{mmol}, 0.05$ equiv.). The reaction was stirred at $\mathrm{rt}$ for $16 \mathrm{~h}$, after which DCM $(10 \mathrm{~mL})$ was added. The mixture was washed with aq. EDTA $(10 \mathrm{mg} / \mathrm{mL}, 10 \mathrm{~mL})$, brine $(2 \times 10 \mathrm{~mL})$, dried over $\mathrm{Na}_{2} \mathrm{SO}_{4}$ and concentrated under reduced pressure. The resulting residue was purified by flash chromatography (silica gel, 9/1 EtOAc/hexane) to provide the desired product as a white solid (61 mg, 98\%). Method B: To a solution of 5-(1-benzyl-1H-1,2,3-triazol-4-yl)- $N$-(1((triisopropylsilyl)ethynyl)-1H-benzo[d]imidazol-6-yl)pentanamide $(20 \mathrm{mg}, 0.051 \mathrm{mmol}, 1$ equiv.) in $\mathrm{MeOH} / \mathrm{H}_{2} \mathrm{O}(1 / 1,2 \mathrm{~mL})$ was added benzyl azide $(7 \mu \mathrm{L}, 0.051 \mathrm{mmol}, 1$ equiv.), AMTC (1 mg, 0.0051 mmol, 0.1 equiv.), $\mathrm{Cu}(\mathrm{OAc})_{2}(0.5 \mathrm{mg}, 0.0026 \mathrm{mmol}, 0.05$ equiv. $)$ and $\mathrm{NaAsc}(1 \mathrm{mg}, 0.0026 \mathrm{mmol}, 0.1$ equiv.). The reaction was stirred at $\mathrm{rt}$ for $16 \mathrm{~h}$, after which DCM $(10 \mathrm{~mL})$ was added. The mixture was washed with aq. EDTA $(10 \mathrm{mg} / \mathrm{mL}, 10 \mathrm{~mL})$, brine $(2 \times 10 \mathrm{~mL})$, dried over $\mathrm{Na}_{2} \mathrm{SO}_{4}$ and concentrated under reduced pressure. The resulting residue was purified by flash chromatography (silica gel, 9/1 EtOAc/hexane) to provide the desired product as a white solid (25 mg, 94\%). ${ }^{1} \mathrm{H}$ NMR $\left(\mathrm{CDCl}_{3}, 400\right.$ MHz,): $\delta 8.23$ (s, 1H), 8.40 (br. s, 1H), 8.38 (s, 1H), 7.92 (s, 1H), 7.68 (d, J= $7.5 \mathrm{~Hz}, 1 \mathrm{H}), 7.33-7.30$ (m, 4H), 7.23-7.20 (m, 3H), $5.45(\mathrm{~s}, 2 \mathrm{H}), 4.47(\mathrm{tt}, J=11.8,3.8 \mathrm{~Hz}, 1 \mathrm{H}), 2.69(\mathrm{t}, \mathrm{J}=6.5 \mathrm{~Hz}, 2 \mathrm{H}), 2.43$ (t, $J=6.7 \mathrm{~Hz}, 2 \mathrm{H}), 2.25(\mathrm{dd}, J=12.7,2.4 \mathrm{~Hz}, 2 \mathrm{H}), 1.95-1.89(\mathrm{~m}, 2 \mathrm{H}), 1.84(\mathrm{dd}, J=12.1,3.6 \mathrm{~Hz}, 2 \mathrm{H})$, 
1.79-1.70 (m, 6H), $1.47(\mathrm{tt}, J=12.9,3.5 \mathrm{~Hz}, 2 \mathrm{H}) .{ }^{13} \mathrm{C} \mathrm{NMR}\left(\mathrm{CDCl}_{3}, 100 \mathrm{MHz}\right): \delta 172.0,135.5$, $134.9,129.2,128.8,128.1,121.1,120.6,116.3,112.3,102.4,61.3,54.1,37.1,33.4,28.6,25.3,26.2$, 25.1, 25.0. 5 signals not observed/coincident. IR $v_{\max }$ (neat): 3257, 3125, 3062, 2922, 2852, 2093, $1671,1584,1547,1496,1485,1446,1299,1216,1050,816,799,729 \mathrm{~cm}^{-1}$. HRMS (ESI-TOF) $\mathrm{m} / z$ : $[\mathrm{M}+\mathrm{Na}]^{+}$Calcd for $\mathrm{C}_{29} \mathrm{H}_{33} \mathrm{~N}_{9} \mathrm{ONa}$ 546.2700; Found 546.2660.

N-(1-(1-Benzyl-1H-1,2,3-triazol-4-yl)-1H-benzo[d]imidazol-6-yl)-5-(1-cyclohexyl-1H-1,2,3-triazol-4yl)pentanamide (11b). Method A: To a solution of 5-(1-cyclohexyl-1H-1,2,3-triazol-4-yl)-N-(1((triisopropylsilyl)ethynyl)-1H-benzo[d]imidazol-6-yl)pentanamide (30 mg, $0.06 \mathrm{mmol}, 1$ equiv.) in MeCN ( $1 \mathrm{~mL})$ was added benzyl azide $(7 \mu \mathrm{L}, 0.06 \mathrm{mmol}, 1$ equiv.), TBAF ( $20 \mu \mathrm{L}, 0.07 \mathrm{mmol}, 1.1$ equiv.) and $\mathrm{Cu}(\mathrm{OAc})_{2}(0.5 \mathrm{mg}, 0.003 \mathrm{mmol}, 0.05$ equiv. $)$. The reaction was stirred at $\mathrm{rt}$ for $2 \mathrm{~h}$, after which DCM $(10 \mathrm{~mL})$ was added. The mixture was washed with aq. EDTA $(10 \mathrm{mg} / \mathrm{mL}, 10 \mathrm{~mL})$, brine ( $2 \times 10 \mathrm{~mL}$ ), dried over $\mathrm{Na}_{2} \mathrm{SO}_{4}$ and concentrated under reduced pressure. The resulting residue was purified by flash chromatography (silica gel, 9/1 EtOAc/hexane) to provide the desired product as a white solid (30 mg, 95\%). Method B: To a solution of $N$-(1-(1-benzyl-1H-1,2,3-triazol-4-yl)-1Hbenzo[d]imidazol-6-yl)hept-6-ynamide (30 mg, $0.08 \mathrm{mmol}, 1$ equiv.) in $\mathrm{MeOH} / \mathrm{H}_{2} \mathrm{O}(1 / 1,2 \mathrm{~mL}$ ) was added cyclohexylazide (10 mg, $0.08 \mathrm{mmol}, 1$ equiv.), AMTC (1.6 mg, $0.008 \mathrm{mmol}, 0.1$ equiv.), $\mathrm{Cu}(\mathrm{OAc})_{2}(0.8 \mathrm{mg}, 0.004 \mathrm{mmol}, 0.05$ equiv.) and $\mathrm{NaAsc}(3 \mathrm{mg}, 0.008 \mathrm{mmol}, 0.1$ equiv.). The reaction was stirred at $\mathrm{rt}$ for $24 \mathrm{~h}$, after which DCM $(10 \mathrm{~mL})$ was added. The mixture was washed with aq. EDTA $(10 \mathrm{mg} / \mathrm{mL}, 10 \mathrm{~mL})$, brine $(2 \times 10 \mathrm{~mL})$, dried over $\mathrm{Na}_{2} \mathrm{SO}_{4}$ and concentrated under reduced pressure. The resulting residue was purified by flash chromatography (silica gel, 9/1 EtOAc/hexane) to provide the desired product as a yellow solid (30 mg, 71\%). ${ }^{1} \mathrm{H}$ NMR $\left(\mathrm{CDCl}_{3}, 400 \mathrm{MHz}\right.$,): $\delta 8.67$ (br. s, 1H), 8.10 (br.s, 1H), 7.88 (br. s, 1H), 7.41-7.38 (m, 6H), 7.23 (m, 2H), $5.65(\mathrm{~s}, 2 \mathrm{H}), 3.24$ (m, 2H), $2.48(\mathrm{t}, J=5.8 \mathrm{~Hz}, 2 \mathrm{H}), 2.29-2.26(\mathrm{~m}, 1 \mathrm{H}), 2.20-2.18(\mathrm{~m}, 2 \mathrm{H}), 1.90-1.75(\mathrm{~m}, 6 \mathrm{H}), 1.69-1.60(\mathrm{~m} 4 \mathrm{H})$, 1.45-1.42 (m, 2H). ${ }^{13} \mathrm{C}$ NMR ( $\left.\mathrm{CDCl}_{3}, 100 \mathrm{MHz}\right): \delta$ 129.4, 129.2, 128.3, 124.6, 31.6, 30.3, 29.8, 29.6, 28.0, 25.3, 24.8, 24.5, 20.0, 13.9. 15 signals not observed/coincident. IR $v_{\max }$ (neat): 3300, 2954, 2928, $2855,1736,1677,1628,1600,1587,1548,1496,1441,1455,1379,1364,1301,1234,864,821,725$ $\mathrm{cm}^{-1}$. HRMS (ESI-TOF): m/z: [M + Na $]^{+}$Calcd for $\mathrm{C}_{29} \mathrm{H}_{33} \mathrm{~N}_{9} \mathrm{ONa}$ 546.2700; Found 546.2689. 
2-((4-(p-Tolyl)-1H-1,2,3-triazol-1-yl)methyl)pyridine (12). ${ }^{33}$ To a solution of 5,6-dimethyl-1((triisopropylsilyl)ethynyl)-1H-benzo[d]imidazole 1 (49 mg, $0.15 \mathrm{mmol}, 1$ equiv.) and 1-ethynyl-4methylbenzene (19 $\mu \mathrm{L}, 0.15 \mathrm{mmol}, 1$ equiv.) in $\mathrm{MeCN}(0.75 \mathrm{~mL})$ was added 2-(azidomethyl)pyridine (20 mg, 0.15 mmol, 1 equiv.), (azidomethyl)benzene (19 $\mu \mathrm{L}, 0.15 \mathrm{mmol}, 1$ equiv.), and $\mathrm{Cu}(\mathrm{OAc})_{2}(1.4$ $\mathrm{mg}, 0.0075 \mathrm{mmol}, 0.05$ equiv.). The reaction was stirred at $\mathrm{rt}$ for $5 \mathrm{~h}$ before being filtered through celite and concentrated under reduced pressure. The crude mixture was dissolved in $\mathrm{MeCN}(0.75 \mathrm{~mL})$ before adding $\mathrm{Cu}(\mathrm{OAc})_{2}(1.4 \mathrm{mg}, 0.0075 \mathrm{mmol}, 0.05$ equiv.) and TBAF (1 M in THF, $22.5 \mu \mathrm{L}, 0.225$ mmol, 1.5 equiv.). The reaction was stirred at $\mathrm{rt}$ for a further $16 \mathrm{~h}$, after which EtOAc $(10 \mathrm{~mL})$ was added. The mixture was washed with aq. EDTA $(10 \mathrm{mg} / \mathrm{mL}, 10 \mathrm{~mL})$, brine $(2 \times 10 \mathrm{~mL})$, dried over $\mathrm{Na}_{2} \mathrm{SO}_{4}$, and concentrated under reduced pressure. The resulting residue was purified by flash chromatography (silica gel, $\mathrm{Et}_{2} \mathrm{O} / \mathrm{MeOH} / \mathrm{NEt}_{3} 20 / 1 / 1$ ) to provide the desired product as a white solid (32 mg, 85\%). ${ }^{1} \mathrm{H}$ NMR $\left(\mathrm{CDCl}_{3}, 500 \mathrm{MHz}\right): \delta 8.61$ (br. s, 1H), 7.89 (s, 1H), $7.72(\mathrm{~s}, 1 \mathrm{H}), 7.71(\mathrm{~s}, 1 \mathrm{H})$, $7.68(\mathrm{td}, J=7.6,1.4 \mathrm{~Hz}, 1 \mathrm{H}), 7.26(\mathrm{t}, J=5.4 \mathrm{~Hz}, 1 \mathrm{H}), 7.22(\mathrm{t}, J=5.6 \mathrm{~Hz}, 3 \mathrm{H}), 5.69(\mathrm{~s}, 2 \mathrm{H}), 2.36(\mathrm{~s}$, $3 \mathrm{H}) .{ }^{13} \mathrm{C} \mathrm{NMR}\left(\mathrm{CDCl}_{3}, 100 \mathrm{MHz}\right): \delta 154.7,149.8,148.5,138.1,137.5,129.6,127.8,125.7,123.6$, 122.6, 120.0, 55.8, 21.4 .

\section{ACKNOWLEDGMENTS}

M.Z.C.H. thanks the University of Strathclyde for a PhD scholarship. C.P.S. thanks the Carnegie Trust for the Universities of Scotland for a PhD scholarship. We thank the EPSRC UK National Mass Spectrometry Facility at Swansea University for analyses.

\section{ASSOCIATED CONTENT}

Supporting information. Data for optimization, NMR and IR spectra for characterization.

\section{REFERENCES}

(1) For seminal reports, see: a) Kolb, H. C.; Finn, M. G.; Sharpless, K. B. Angew. Chem. Int. Ed. 2001, 40, 2004-2021; b) Tornøe, C. W.; Christensen, C.; Meldal, M. J. Org. Chem. 2002, 67, 3057-3064. 
(2) For reviews see: a) Hein, J. E.; Fokin, V. V. Chem. Soc. Rev. 2010, 39, 1302-1315; b) Meldal, M.; Tornøe, C. W. Chem. Rev. 2008, 108, 2952-3015; c) Moses, J. E.; Moorhouse, A. D. Chem. Soc. Rev. 2007, 36, 1249-1262.

(3) Weixian, X.; Scott, T. F.; Kloxin, C. J.; Bowman, C. N. Adv. Funct. Mater. 2014, 24, 2572-2590.

(4) Thirumurugan, P.; Matosiuk, D.; Jozwiak, K. Chem. Rev. 2013, 113, 4905-4979.

(5) Castro, V.; Rodríguez, H.; Albericio, F. ACS Comb. Sci. 2016, 18, 1-14.

(6) a) Wang, Z.; Li, Y.; Dong, X.-H.; Yu, X.; Guo, K.; Su, H.; Yue, K.; Wesdemiotis, C.; Cheng, S. Z. D.; Zhang, W. B. Chem. Sci. 2013, 4, 1345-1352; b) Kele, P.; Mezö, G.; Achatz, D.; Wolfbeis, O. S. Angew. Chem. Int. Ed. 2009, 48, 344-347; c) DeForest, C. A.; Polizzotti, B. D.; Anseth, K. S. Nature Materials 2009, 8, 659-664.

(7) a) Yuan, Z.; Kuang, G. C.; Clark, R. J.; Zhu, L. Org. Lett. 2012, 14, 2590-2593; b) Kuang, G. C.; Guha, P. M.; Brotherton, W. S.; Simmons, J. T.; Stankee, L. A.; Nguyen, B. T.; Clark, R. J.; Zhu, L. J. Am. Chem. Soc. 2011, 133, 13984-14001. (c) Urankar, D.; Steinbücher, M.; Kosjek, J.; Košmrlj, J. Tetrahedron 2010, 66, 2602-2613.

(8) a) Gramlich, P. M. E.; Warncke, S.; Geirlich, J.; Carell, T. Angew. Chem. Int. Ed. 2008, 47, 34423444; b) Aucagne, V.; Leigh, D. A. Org. Lett. 2006, 8, 4505-4507.

(9) a) Kislukhin, A. A.; Hong, V. P.; Breitenkamp, K. E.; Finn, M. G. Bioconjugate Chem. 2013, 24, 684-689; b) Zhang, X.; Li, H.; You, L.; Tang, Y.; Hsung, R. P. Adv. Synth. Catal. 2006, 348, 2437-2442; c) Zhang, X.; Hsung, R. P.; You, L. Org. Biomol. Chem. 2006, 4, 2679-2682.

(10) a) Beal, D. M. Albrow, V. E.; Burslem, G.; Hitchen, L.; Fernandes, C.; Lapthorn, C.; Roberts, L. R.; Selby, M. D.; Jones, L. H. Org. Biomol. Chem. 2012, 10, 548-554; b) Agard, N. J.; Prescher, J. A.; Bertozzi, C. R. J. Am. Chem. Soc. 2004, 126, 15046-15047.

(11) Yoshida, S.; Hatakeyama, Y.; Johmoto, K.; Uekusa, H.; Hosoya, T. J. Am. Chem. Soc. 2014, 136, $13590-13593$.

(12) Tummatorn, J.; Batsomboon, P.; Clark, R. J.; Alabugin, I. V.; Dudley, G. B. J. Org. Chem. 2012, 77, 2093-2097.

(13) a) Münster, N.; Nikodemiak, P.; Koert, U. Org. Lett. 2016, 18, 4296-4299; b) Ramsubhag, R. R.; Dudley, G. B. Org. Biomol. Chem. 2016, 14, 5028-5031. 
(14) Seath, C. P.; Burley, G. A.; Watson, A. J. B. Angew. Chem. Int. Ed. 2017, 56, 3314-3318.

(15) Hatit, M. Z. C.; Sadler, J. C.; McLean, L. A.; Whitehurst, B. C.; Seath, C. P.; Humphreys, L. D.; Young, R. J.; Watson, A. J. B.; Burley, G. A. Org. Lett. 2016, 18, 1694-1697.

(16) Burley, G. A.; Davies, D. L.; Griffith, G. A.; Lee, M.; Singh, K. J. Org. Chem. 2010, 75, 980983.

(17) Belanger, G.; Dupuis, M.; Larouche-Gauthier, R. J. Org. Chem. 2012, 77, 3215-3221.

(18) Capella, L.; Montevecchi, P. C.; Nanni, D. J. Org. Chem. 1994, 59, 3368-3374.

(19) Sahoo, L.; Singhamahapatra, A.; Loganathan, D. Org. Biomol. Chem. 2014, 12, 2615-2625.

(20) Khedar, P.; Pericherla, K.; Kumar, A. Synlett 2014, 25, 515-518.

(21) Schmitz, J.; Li, T.; Bartz, U.; Gütschow, M. ACS Med. Chem. Lett. 2016, 7, 211-216.

(22) Mamidyala, S. K.; Cooper, M. A. Chem. Commun. 2013, 49, 8407-8409.

(23) Sivakumar, K.; Xie, F.; Cash, B. M.; Long, S.; Barnhill, H. N.; Wang, Q. Org. Lett. 2004, 6, 4603-4606.

(24) Wolfram, S.; Wurfel, H.; Habenicht, S. H.; Lembke, C.; Richter, P.; Birckner, E.; Beckert, R.; Pohnert, G. Beilstein J. Org. Chem. 2014, 10, 2470-2479.

(25) Huang, B.; Kukowska-Latallo, J. F.; Tang, S.; Zong, H.; Johnson, K. B.; Desai, A.; Gordon, C. L.; Leroueil, P. R.; Baker, J. R. Bioorg. Med. Chem. Lett. 2012, 22, 3152-3156.

(26) Oakdale, J. S.; Sit, R. K.; Fokin, V. V. Chem. Eur. J. 2014, 20, 11101-11110.

(27) Chen, Y.-L.; Sharma, P.; Liu, R.-S. Chem. Commun. 2016, 52, 3187-3190.

(28) Szafranski, P. W.; Kasza, P.; Cegła, M. T. Tetrahedron Lett. 2015, 56, 6244-6247.

(29) Zhang, X.; Hsung, R. P.; You, L. Org. Biomol. Chem. 2006, 4, 2679-2682.

(30) Tale, R. H.; Gopula, V. B.; Toradmal, G. K. Tetrahedron Lett. 2015, 56, 5864-5869.

(31) Zheng, Z.; Shi, L. Tetrahedron Lett. 2016, 57, 5132-5134.

(32) Taher, A.; Nandi, D.; Islam, R. U. 1.; Choudhary, M.; Mallick, K. RSC Adv. 2015, 5, 4727547283

(33) Bolje, A.; Urankar, D.; Kosmrlj, J. Eur. J. Org. Chem. 2014, 36, 8167-8181. 
TOC GRAPHIC

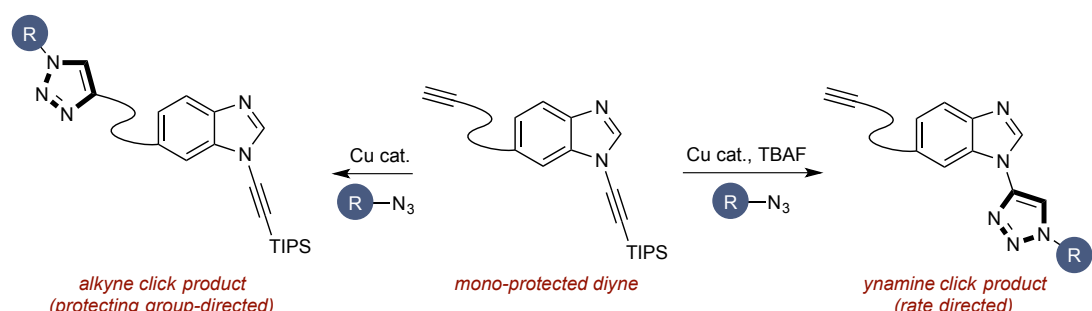

(protecting group-directed)

mono-protected diyne

(rate directed) 